JOURNAL OF THE

AMERICAN MATHEMATICAL SOCIETY

Volume 25, Number 1, January 2012, Pages 1-20

S $0894-0347(2011) 00713-3$

Article electronically published on July 8, 2011

\title{
EQUIVALENCES BETWEEN FUSION SYSTEMS OF FINITE GROUPS OF LIE TYPE
}

\author{
CARLES BROTO, JESPER M. MØLLER, AND BOB OLIVER
}

When $G$ is a finite group and $p$ is a prime, the fusion system $\mathcal{F}_{p}(G)$ is the category whose objects are the $p$-subgroups of $G$, and whose morphisms are the homomorphisms between subgroups induced by conjugation in $G$. If $G^{\prime}$ is another finite group, then $\mathcal{F}_{p}(G)$ and $\mathcal{F}_{p}\left(G^{\prime}\right)$ are isotypically equivalent if there is an equivalence of categories between them which commutes, up to natural isomorphism of functors, with the forgetful functors from $\mathcal{F}_{p}(-)$ to the category of groups. Alternatively, $\mathcal{F}_{p}(G)$ and $\mathcal{F}_{p}\left(G^{\prime}\right)$ are isotypically equivalent if there is an isomorphism between Sylow $p$-subgroups of $G$ and of $G^{\prime}$ which is "fusion preserving" in the sense of Definition 1.2 below.

The goal of this paper is to use methods from homotopy theory to prove that certain pairs of fusion systems of finite groups of Lie type are isotypically equivalent. Our main result is the following theorem.

Theorem A. Fix a prime $p$, a connected reductive group scheme $\mathbb{G}$ over $\mathbb{Z}$, and a pair of prime powers $q$ and $q^{\prime}$ both prime to $p$. Then the following hold, where “ $\simeq$ always means isotypically equivalent.

(a) $\mathcal{F}_{p}(\mathbb{G}(q)) \simeq \mathcal{F}_{p}\left(\mathbb{G}\left(q^{\prime}\right)\right)$ if $\overline{\langle q\rangle}=\overline{\left\langle q^{\prime}\right\rangle}$ as subgroups of $\mathbb{Z}_{p}^{\times}$.

(b) If $\mathbb{G}$ is of type $A_{n}, D_{n}$, or $E_{6}$, and $\tau$ is a graph automorphism of $\mathbb{G}$, then $\mathcal{F}_{p}\left({ }^{\tau} \mathbb{G}(q)\right) \simeq \mathcal{F}_{p}\left({ }^{\tau} \mathbb{G}\left(q^{\prime}\right)\right)$ if $\overline{\langle q\rangle}=\overline{\left\langle q^{\prime}\right\rangle}$ as subgroups of $\mathbb{Z}_{p}^{\times}$.

(c) If the Weyl group of $\mathbb{G}$ contains an element which acts on the maximal torus by inverting all elements, then $\mathcal{F}_{p}(\mathbb{G}(q)) \simeq \mathcal{F}_{p}\left(\mathbb{G}\left(q^{\prime}\right)\right)\left(\right.$ or $\mathcal{F}_{p}\left({ }^{\tau} \mathbb{G}(q)\right) \simeq \mathcal{F}_{p}\left({ }^{\tau} \mathbb{G}\left(q^{\prime}\right)\right)$ for $\tau$ as in (b)) if $\overline{\langle-1, q\rangle}=\overline{\left\langle-1, q^{\prime}\right\rangle}$ as subgroups of $\mathbb{Z}_{p}^{\times}$.

(d) If $\mathbb{G}$ is of type $A_{n}, D_{n}$ for $n$ odd, or $E_{6}$, and $\tau$ is a graph automorphism of $\mathbb{G}$ of order two, then $\mathcal{F}_{p}\left({ }^{\tau} \mathbb{G}(q)\right) \simeq \mathcal{F}_{p}\left(\mathbb{G}\left(q^{\prime}\right)\right)$ if $\overline{\langle-q\rangle}=\overline{\left\langle q^{\prime}\right\rangle}$ as subgroups of $\mathbb{Z}_{p}^{\times}$.

Here, in all cases, $\mathbb{G}(q)$ means the fixed subgroup of the field automorphism $\psi^{q}$ acting on $\mathbb{G}\left(\overline{\mathbb{F}}_{q}\right)$, and ${ }^{\tau} \mathbb{G}(q)$ means the fixed subgroup of $\tau \psi^{q}$ acting on $\mathbb{G}\left(\overline{\mathbb{F}}_{q}\right)$.

Received by the editors March 17, 2010 and, in revised form, June 14, 2011.

2010 Mathematics Subject Classification. Primary 20D06; Secondary 55R37, 20D20.

Key words and phrases. groups of Lie type, fusion systems, classifying spaces, $p$-completion.

The first author is partially supported by FEDER-MICINN grant MTM 2010-20692.

The second author was partially supported by the Danish National Research Foundation (DNRF) through the Centre for Symmetry and Deformation.

The third author was partially supported by UMR 7539 of the CNRS, and by project ANR BLAN08-2_338236, HGRT. 
Note that we are comparing the fusion of groups of Lie type with different defining characterisitics. Thus Theorem A gives many examples of pairs of groups which all have the equivalent $p$-fusion systems, but where these equivalences are not induced by any homomorphisms between the groups. This seems to be one reason why there is not yet a purely algebraic proof of these results.

We remark here that this theorem does not apply when comparing fusion systems of $S O_{n}^{ \pm}(q)$ and $S O_{n}^{ \pm}\left(q^{\prime}\right)$ for even $n$, at least not when $q$ or $q^{\prime}$ is a power of 2 , since $S O_{n}(K)$ is not connected when $K$ is algebraically closed of characteristic two. Instead, one must compare the groups $\Omega_{n}^{ \pm}(-)$. For example, for even $n \geq 4, \Omega_{n}^{+}(4)$ and $\Omega_{n}^{+}(7)$ have equivalent 3-fusion systems, while $S O_{n}^{+}(4)$ and $S O_{n}^{+}(7)$ do not.

Points (a)-(c) of Theorem $\mathrm{A}$ will be proven in Proposition 3.2, where we deal with the more general situation where $\mathbb{G}$ is reductive (thus including cases such as $\mathbb{G}=G L_{n}$ ). Point (d) will be proven as Proposition 3.3. In all cases, this will be done by showing that the $p$-completed classifying spaces of the two groups are homotopy equivalent. A theorem of Martino and Priddy (Theorem 1.5 below) then implies that the fusion systems are isotypically equivalent.

Since $p$-completion of spaces plays a central role in our proofs, we give a very brief outline here of what it means and refer to the book of Bousfield and Kan BK] for more details. They define $p$-completion as a functor from spaces to spaces, which we denote $(-)_{p}^{\wedge}$ here, and this functor comes with a map $X \stackrel{\kappa_{p}(X)}{\longrightarrow} X_{p}^{\wedge}$, which is natural in $X$. For any map $f: X \longrightarrow Y, f_{p}^{\wedge}$ is a homotopy equivalence if and only if $f$ is a $\bmod p$ equivalence; i.e., $H^{*}\left(f ; \mathbb{F}_{p}\right)$ is an isomorphism from $H^{*}\left(Y ; \mathbb{F}_{p}\right)$ to $H^{*}\left(X ; \mathbb{F}_{p}\right)$. A space $X$ is called " $p$-good" if $\kappa_{p}\left(X_{p}^{\wedge}\right)$ is a homotopy equivalence (equivalently, $\kappa_{p}(X)$ is a $\bmod p$ equivalence). In particular, all spaces with finite fundamental group are $p$-good. If $X$ is $p$-good, then $\kappa_{p}(X): X \longrightarrow X_{p}^{\wedge}$ is universal among all mod $p$ equivalences $X \longrightarrow Y$. If $X$ and $Y$ are both $p$-good, then $X_{p}^{\wedge} \simeq Y_{p}^{\wedge}$ (the $p$-completions are homotopy equivalent) if and only if there is a third space $Z$, and $\bmod p$ equivalences $X \longrightarrow Z \longleftarrow Y$.

By a theorem of Friedlander (stated as Theorem 3.1 below), $B\left({ }^{\tau} \mathbb{G}(q)\right)_{p}^{\wedge}$ is the homotopy fixed space (Definition 2.1) of the action of $\tau \psi^{q}$ on $B \mathbb{G}(\mathbb{C})_{p}^{\wedge}$. By our Theorem 2.4 if $X$ is a $p$-complete space which satisfies certain technical conditions, then for any pair of self-equivalences $\alpha$ and $\beta$ such that $\overline{\langle\alpha\rangle}=\overline{\langle\beta\rangle}$ in $\operatorname{Out}(X)$, $X^{h \alpha} \simeq X^{h \beta}$. Here, $\operatorname{Out}(X)$ is the group of homotopy classes of self-equivalences of $X$, equipped with a certain " $p$-adic" topology. The groups $\operatorname{Out}\left(B \mathbb{G}(\mathbb{C})_{p}^{\wedge}\right)$ were described precisely by Jackowski, McClure, and Oliver in [JMO, and Theorem A follows upon combining these results.

Corresponding results for the Suzuki and Ree groups can also be shown using this method of proof. But since there are much more elementary proofs of these results (all equivalences are induced by inclusions of groups), and since it seemed difficult to find a nice formulation of the theorem which included everything, we decided to leave them out of the statement.

As another application of these results, we prove that for any prime $p$ and any prime power $q \equiv 1(\bmod p)$, the fusion systems $\mathcal{F}_{p}\left(G_{2}(q)\right)$ and $\mathcal{F}_{p}\left({ }^{3} D_{4}(q)\right)$ are isotypically equivalent if $p \neq 3$, and the fusion systems $\mathcal{F}_{p}\left(F_{4}(q)\right)$ and $\mathcal{F}_{p}\left({ }^{2} E_{6}(q)\right)$ are isotypically equivalent if $p \neq 2$ (Example 4.4).

It seems likely that Theorem $\mathrm{A}$ can also be shown directly using a purely algebraic proof, but the people we have asked do not know of one, and there does not seem to be any in the literature. There is a very closely related result by Michael Larsen 
GR, Theorem A.12], which implies that two Chevalley groups $\mathbb{G}(K)$ and $\mathbb{G}\left(K^{\prime}\right)$ over algebraically closed fields of characteristic prime to $p$ have equivalent $p$-fusion systems when defined appropriately for these infinite groups. There are standard methods for comparing the finite subgroups of $\mathbb{G}\left(\overline{\mathbb{F}}_{q}\right)$ (of order prime to $q$ ) with those in its finite Chevalley subgroups, but we have been unable to get enough control over them to prove Theorem $\mathrm{A}$ using Larsen's theorem.

The paper is organized as follows. In Section 1, we give a general survey of fusion categories of finite groups and their relationship to $p$-completed classifying spaces. Then, in Section 2, we prove a general theorem (Theorem 2.4) comparing homotopy fixed points of different actions on the same space, and apply it in Section 3 to prove Theorem $\mathrm{A}$. In Section 4, we show a second result about homotopy fixed points, which is used to prove the result comparing fusion systems of $G_{2}(q)$ and ${ }^{3} D_{4}(q)$, and $F_{4}(q)$ and ${ }^{2} E_{6}(q)$. At the end, in Appendix A, we prove a few other equivalence relations between fusion systems of classical groups (Proposition A.3) using more elementary methods.

\section{Fusion CATEGORIES}

We begin with a quick summary of what is needed here about fusion systems of finite groups.

Definition 1.1. For any finite group $G$ and any prime $p, \mathcal{F}_{p}(G)$ denotes the category whose objects are the $p$-subgroups of $G$, and where

$$
\operatorname{Mor}_{\mathcal{F}_{p}(G)}(P, Q)=\left\{\varphi \in \operatorname{Hom}(P, Q) \mid \varphi=c_{x} \text { for some } x \in G\right\} .
$$

Here, $c_{x}$ denotes the conjugation homomorphism: $c_{x}(g)=x g x^{-1}$. If $S \in \operatorname{Syl}_{p}(G)$ is a Sylow $p$-subgroup, then $\mathcal{F}_{S}(G) \subseteq \mathcal{F}_{p}(G)$ denotes the full subcategory with objects the subgroups of $S$.

A functor $F: \mathcal{C} \longrightarrow \mathcal{C}^{\prime}$ is an equivalence of categories if it induces bijections on isomorphism classes of objects and on all morphism sets. This is equivalent to the condition that there be a functor from $\mathcal{C}^{\prime}$ to $\mathcal{C}$ such that both composites are naturally isomorphic to the identity. An inclusion of a full subcategory is an equivalence if and only if every object in the larger category is isomorphic to some object in the smaller one. Thus when $G$ is finite and $S \in \operatorname{Syl}_{p}(G)$, the inclusion $\mathcal{F}_{S}(G) \subseteq \mathcal{F}_{p}(G)$ is an equivalence of categories by the Sylow theorems.

In general, we write $\Psi: \mathcal{C}_{1} \stackrel{\cong}{\longrightarrow} \mathcal{C}_{2}$ to mean that $\Psi$ is an isomorphism of categories (bijective on objects and on morphisms) and write $\Psi: \mathcal{C}_{1} \stackrel{\simeq}{\longrightarrow} \mathcal{C}_{2}$ to mean that $\Psi$ is an equivalence of categories.

In the following definition, for any finite $G, \lambda_{G}$ denotes the forgetful functor from $\mathcal{F}_{p}(G)$ to the category of groups.

Definition 1.2. Fix a prime $p$, a pair of finite groups $G$ and $G^{*}$, and Sylow $p$ subgroups $S \in \operatorname{Syl}_{p}(G)$ and $S^{*} \in \operatorname{Syl}_{p}\left(G^{*}\right)$.

(a) An isomorphism $\varphi: S \stackrel{\cong}{\longrightarrow} S^{*}$ is fusion-preserving if for all $P, Q \leq S$ and $\alpha \in \operatorname{Hom}(P, Q)$,

$$
\alpha \in \operatorname{Mor}_{\mathcal{F}_{p}(G)}(P, Q) \Longleftrightarrow \varphi \alpha \varphi^{-1} \in \operatorname{Mor}_{\mathcal{F}_{p}\left(G^{*}\right)}(\varphi(P), \varphi(Q)) .
$$


(b) An equivalence of categories $T: \mathcal{F}_{p}(G) \longrightarrow \mathcal{F}_{p}\left(G^{*}\right)$ is isotypical if there is a natural isomorphism of functors $\omega: \lambda_{G} \longrightarrow \lambda_{G^{*}} \circ T$, i.e., if there are isomorphisms $\omega_{P}: P \stackrel{\cong}{\longrightarrow} T(P)$ such that $\omega_{Q} \circ \varphi=T(\varphi) \circ \omega_{P}$ for each $\varphi \in$ $\operatorname{Hom}_{G}(P, Q)$.

In other words, in the above situation, an isomorphism $\varphi: S \longrightarrow S^{*}$ is fusionpreserving if and only if it induces an isomorphism from $\mathcal{F}_{S}(G) \stackrel{\cong}{\longrightarrow} \mathcal{F}_{S^{*}}\left(G^{*}\right)$ by sending $P$ to $\varphi(P)$ and $\alpha$ to $\varphi \alpha \varphi^{-1}$. Any such isomorphism of categories extends to an equivalence $\mathcal{F}_{p}(G) \stackrel{\simeq}{\longrightarrow} \mathcal{F}_{p}\left(G^{*}\right)$, which is easily seen to be isotypical. In fact, two fusion categories $\mathcal{F}_{p}(G)$ and $\mathcal{F}_{p}\left(G^{*}\right)$ are isotypically equivalent if and only if there is a fusion-preserving isomorphism between Sylow $p$-subgroups. This is shown in the following proposition, where for any pair of groups $H$ and $G$, we write $\operatorname{Rep}(H, G)=\operatorname{Hom}(H, G) / \operatorname{Inn}(G)$ (the set of conjugacy classes of homomorphisms).

Proposition 1.3. Fix a pair of finite groups $G$ and $G^{*}$, a prime $p$ and Sylow p-subgroups $S \leq G$ and $S^{*} \leq G^{*}$. Then the following are equivalent:

(a) There is a fusion-preserving isomorphism $\varphi: S \stackrel{\cong}{\longrightarrow} S^{*}$.

(b) $\mathcal{F}_{p}(G)$ and $\mathcal{F}_{p}\left(G^{*}\right)$ are isotypically equivalent.

(c) There are bijections $\operatorname{Rep}(P, G) \stackrel{\cong}{\operatorname{Rep}}\left(P, G^{*}\right)$, for all finite p-groups $P$, which are natural in $P$.

Proof. This was essentially shown by Martino and Priddy $\mathrm{MP}$, but not completely explicitly. By the above remarks, (a) implies (b).

$(\mathbf{b} \Longrightarrow \mathbf{c}):$ Fix an isotypical equivalence $T: \mathcal{F}_{p}(G) \longrightarrow \mathcal{F}_{p}\left(G^{*}\right)$, and let $\omega$ be an associated natural isomorphism. Thus $\omega_{P} \in \operatorname{Iso}(P, T(P))$ for each $p$-subgroup $P \leq G$, and $\omega_{Q} \circ \alpha=T(\alpha) \circ \omega_{P}$ for all $\alpha \in \operatorname{Hom}_{G}(P, Q)$. For each $p$-group $Q, \omega$ defines a bijection from $\operatorname{Hom}(Q, G)$ to $\operatorname{Hom}\left(Q, G^{*}\right)$ by sending $\rho$ to $\omega_{\rho(Q)} \circ \rho$. For $\alpha, \beta \in \operatorname{Hom}(Q, G)$, the diagram

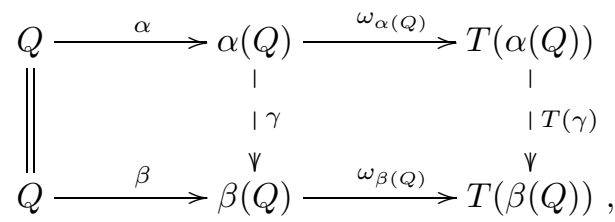

together with the fact that $T$ is an equivalence, proves that $\alpha$ and $\beta$ are $G$-conjugate (there exists $\gamma$ which makes the left-hand square commute) if and only if $\omega_{\alpha(Q)} \circ \alpha$ and $\omega_{\beta(Q)} \circ \beta$ are $G^{*}$-conjugate (there exists $T(\gamma)$ ). Thus $T$ induces bijections $\Phi: \operatorname{Rep}(Q, G) \stackrel{\cong}{\longrightarrow} \operatorname{Rep}\left(Q, G^{*}\right)$, and similar arguments show that $\Phi$ is natural in $Q$.

$(\mathbf{c} \Longrightarrow \mathbf{a}):$ Fix a natural bijection $\Phi: \operatorname{Rep}(-, G) \stackrel{\cong}{\longrightarrow} \operatorname{Rep}\left(-, G^{*}\right)$ of functors on finite $p$-groups. By naturality, $\Phi$ preserves kernels and hence restricts to a bijection between classes of injections. In particular, there are injections of $S$ into $G^{*}$ and $S^{*}$ into $G$, and thus $S \cong S^{*}$. Since conjugation defines a fusion-preserving isomorphism between any two Sylow $p$-subgroups of $G^{*}$, we can assume $S^{*}=\operatorname{Im}\left(\Phi_{S}\left(\operatorname{incl}_{S}^{G}\right)\right)$. Using the naturality of $\Phi$, it is straightforward to check that $\Phi_{S}\left(\operatorname{incl}_{S}^{G}\right)$ is fusionpreserving as an isomorphism from $S$ to $S^{*}$. 
We also note the following, very elementary result about comparing fusion systems.

Proposition 1.4. Fix a prime $p$, and a pair of groups $G_{1}$ and $G_{2}$ such that $\mathcal{F}_{p}\left(G_{1}\right)$ is isotypically equivalent to $\mathcal{F}_{p}\left(G_{2}\right)$. Then the following hold, where " $\simeq$ " always means isotypically equivalent.

(a) If $Z_{i} \leq Z\left(G_{i}\right)$ is central of order prime to $p$, then $\mathcal{F}_{p}\left(G_{i} / Z_{i}\right) \simeq \mathcal{F}_{p}\left(G_{i}\right)$.

(b) If $Z_{1} \leq Z\left(G_{1}\right)$ is a central p-subgroup, and $Z_{2} \leq G_{2}$ is its image under some fusion-preserving isomorphism between Sylow p-subgroups of the $G_{i}$, then $\mathcal{F}_{p}\left(G_{1}\right) \simeq \mathcal{F}_{p}\left(C_{G_{2}}\left(Z_{2}\right)\right)$ and $\mathcal{F}_{p}\left(G_{1} / Z_{1}\right) \simeq \mathcal{F}_{p}\left(C_{G_{2}}\left(Z_{2}\right) / Z_{2}\right)$.

(c) $\mathcal{F}_{p}\left(\left[G_{1}, G_{1}\right]\right) \simeq \mathcal{F}_{p}\left(\left[G_{2}, G_{2}\right]\right)$.

Proof. Points (a) and (b) are elementary. To prove (c), first fix Sylow subgroups $S_{i} \in \operatorname{Syl}_{p}\left(G_{i}\right)$ and a fusion-preserving isomorphism $\varphi: S_{1} \cong S_{2}$. By the focal subgroup theorem (cf. Go, Theorem 7.3.4]), $\varphi\left(S_{1} \cap\left[G_{1}, G_{1}\right]\right)=S_{2} \cap\left[G_{2}, G_{2}\right]$. By BCGLO2, Theorem 4.4], for each $i=1,2$, there is a unique fusion subsystem "of $p$-power index" in $\mathcal{F}_{S_{i}}\left(G_{i}\right)$ over the focal subgroup $S_{i} \cap\left[G_{i}, G_{i}\right]$, which must be the fusion system of $\left[G_{i}, G_{i}\right]$. Hence $\varphi$ restricts to an isomorphism which is fusion-preserving with respect to the commutator subgroups.

Proposition 1.4 implies, for example, that whenever $\mathcal{F}_{p}\left(G L_{n}(q)\right) \simeq \mathcal{F}_{p}\left(G L_{n}\left(q^{\prime}\right)\right)$ for $q$ and $q^{\prime}$ prime to $p$, then there are also equivalences $\mathcal{F}_{p}\left(S L_{n}(q)\right) \simeq \mathcal{F}_{p}\left(S L_{n}\left(q^{\prime}\right)\right)$, $\mathcal{F}_{p}\left(P S L_{n}(q)\right) \simeq \mathcal{F}_{p}\left(P S L_{n}\left(q^{\prime}\right)\right)$, etc.

The following theorem of Martino and Priddy shows that the $p$-fusion in a finite group is determined by the homotopy type of its $p$-completed classifying space. The converse (the Martino-Priddy conjecture) is also true, but the only known proof uses the classification of finite simple groups [01, O2.

Theorem 1.5. Assume $p$ is a prime, and $G$ and $G^{\prime}$ are finite groups, such that $B G_{p}^{\wedge} \simeq B G_{p}^{\prime \wedge}$. Then $\mathcal{F}_{p}(G)$ and $\mathcal{F}_{p}\left(G^{\prime}\right)$ are isotypically equivalent.

Proof. This was shown by Martino and Priddy in $\underline{\mathrm{MP}}$. The key ingredient in the proof is a theorem of Mislin [Ms, pp.457-458], which says that for any finite $p$-group $Q$ and any finite group $G$, there is a bijection

$$
\operatorname{Rep}(Q, G) \underset{B_{p}^{\wedge}}{\cong}\left[B Q, B G_{p}^{\wedge}\right],
$$

where $B_{p}^{\wedge}$ sends the class of a homomorphism $\rho: Q \longrightarrow G$ to the $p$-completion of $B \rho: B Q \longrightarrow B G$. Thus any homotopy equivalence $B G_{p}^{\wedge} \stackrel{\simeq}{\longrightarrow} B G_{p}^{\prime \wedge}$ induces bijections $\operatorname{Rep}(Q, G) \cong \operatorname{Rep}\left(Q, G^{\prime}\right)$, for all $p$-groups $Q$, which are natural in $Q$. The theorem now follows from Proposition 1.3 .

The following proposition will also be useful. When $H \leq G$ is a pair of groups, we regard $\mathcal{F}_{p}(H)$ as a subcategory of $\mathcal{F}_{p}(G)$.

Proposition 1.6. If $H \leq G$ is a pair of groups, then $\mathcal{F}_{p}(H)$ is a full subcategory of $\mathcal{F}_{p}(G)$ if and only if the induced map

$$
\operatorname{Rep}(P, H) \longrightarrow \operatorname{Rep}(P, G)
$$

is injective for all finite p-groups $P$. 
Proof. Assume that $\operatorname{Rep}(P, H)$ injects into $\operatorname{Rep}(P, G)$ for all $P$. For each pair of $p$ subgroups $P, Q \leq H$ and each $\varphi \in \operatorname{Hom}_{G}(P, Q)$, $\left[\operatorname{incl}_{P}^{G}\right]=\left[\operatorname{incl}_{Q}^{G} \circ \varphi\right]$ in $\operatorname{Rep}(P, G)$, so $\left[\operatorname{incl}_{P}^{H}\right]=\left[\operatorname{incl}_{Q}^{H} \circ \varphi\right]$ in $\operatorname{Rep}(P, H)$, and thus $\varphi \in \operatorname{Hom}_{H}(P, Q)$. This proves that $\mathcal{F}_{p}(H)$ is a full subcategory of $\mathcal{F}_{p}(G)$.

Conversely, assume that $\mathcal{F}_{p}(H)$ is a full subcategory. Fix a finite $p$-group $P$, and $\alpha, \beta \in \operatorname{Hom}(P, H)$ such that $[\alpha]=[\beta]$ in $\operatorname{Rep}(P, G)$. Let $\varphi \in \operatorname{Hom}_{G}(\alpha(P), \beta(P))$ be such that $\varphi \circ \alpha=\beta$. Then $\varphi \in \operatorname{Hom}_{H}(\alpha(P), \beta(P))$ since $\mathcal{F}_{p}(H)$ is a full subcategory, and so $[\alpha]=[\beta]$ in $\operatorname{Rep}(P, H)$. This proves injectivity.

Our goal in the next three sections is to construct isotypical equivalences between fusion systems of finite groups at a prime $p$ by constructing homotopy equivalences between their $p$-completed classifying spaces.

\section{2. Номоторy FIXED POINTS OF HOMOtopy SELF-EQUiVALENCES}

We start by defining homotopy orbit spaces and homotopy fixed spaces for a homotopy self-equivalence of a space, i.e., for a homotopy action of the group $\mathbb{Z}$. As usual, $I$ denotes the unit interval $[0,1]$.

Definition 2.1. Fix a space $X$, and a homotopy equivalence $\alpha: X \longrightarrow X$.

(a) The homotopy fixed point set $X^{h \alpha}$ is the space of all continuous maps $\gamma: I \longrightarrow X$ such that $\gamma(1)=\alpha(\gamma(0))$ (with the compact-open topology).

(b) The homotopy orbit space or mapping torus of $\alpha$ is the space $X_{h \alpha}=(X \times I) / \sim$, where $(x, 0) \sim(\alpha(x), 1)$ for all $x \in X$.

(c) Define $p_{\alpha}: X_{h \alpha} \longrightarrow S^{1}$ via projection to $I$, where we identify $S^{1}$ with $I /(0 \sim$ 1).

We can identify the ordinary fixed set of $\alpha$ as the subspace of constant paths in $X^{h \alpha}$. We will show in Lemma B.1 that in contrast to the usual fixed set, $X^{h \alpha}$ is homotopy invariant: its homotopy type depends only on $X$ up to homotopy equivalence and on $\alpha$ up to homotopy.

Remark 2.2. When $\alpha$ is a homeomorphism, then $X_{h \alpha} \stackrel{p_{\alpha}}{\longrightarrow} S^{1}$ is a locally trivial fiber bundle, and $X^{h \alpha}$ is the space of its sections. Conversely, every locally trivial fiber bundle $E \stackrel{p}{\longrightarrow} S^{1}$ with fiber $X$ is homeomorphic to $X_{h \alpha} \stackrel{p_{\alpha}}{\longrightarrow} S^{1}$ for some homeomophism $\alpha$ of $X$ in the homotopy class determined by the action of the generator of $\pi_{1}\left(S^{1}\right)$ on $X$.

When $\alpha$ is a homotopy equivalence but not a homeomorphism, we can arrange to consider $X^{h \alpha}$ as a space of sections by replacing $X$ by the double mapping telescope of $\alpha$. This is the space

$$
\operatorname{Tel}(\alpha)=(X \times I \times \mathbb{Z}) / \sim, \quad \text { where }(x, 0, n) \sim(\alpha(x), 1, n-1) \quad \forall x \in X, n \in \mathbb{Z} .
$$

There is a natural "inclusion" $i$ of $X$ into $\operatorname{Tel}(\alpha)$, defined by setting $i(x)=[x, 0,0]$. This inclusion is a homotopy equivalence (see Lemma B.2).

Let $\widehat{\alpha}: \operatorname{Tel}(\alpha) \longrightarrow \operatorname{Tel}(\alpha)$ be the homeomorphism $\widehat{\alpha}([x, t, n])=[x, t, n+1]$. Then $\widehat{\alpha} \circ i \simeq i \circ \alpha$, so $X^{h \alpha} \simeq \operatorname{Tel}(\alpha)^{h \widehat{\alpha}}$ by Lemma B.1

Remark 2.3. The homotopy fixed point space $X^{\text {h } \alpha}$ of a homotopy equivalence $\alpha$ can also be described as the homotopy pullback of the maps

$$
X \stackrel{\Delta}{\longrightarrow} X \times X \stackrel{(\mathrm{Id}, \alpha)}{\longleftarrow} X,
$$


where $\Delta$ is the diagonal map $\Delta(x)=(x, x)$. In other words, $X^{h \alpha}$ is the space of triples $\left(x_{1}, x_{2}, \phi\right)$, where $x_{1}, x_{2} \in X$, and $\phi$ is a path in $X \times X$ from $\Delta\left(x_{1}\right)=\left(x_{1}, x_{1}\right)$ to $\left(x_{2}, \alpha\left(x_{2}\right)\right)$. Thus $\phi$ is a pair of paths in $X$, one from $x_{1}$ to $x_{2}$ and the other from $x_{1}$ to $\alpha\left(x_{2}\right)$, and these two paths can be composed to give a single path from $x_{2}$ to $\alpha\left(x_{2}\right)$ which passes through the (arbitrary) point $x_{1}$. Hence this definition is equivalent to the one given above. We will come back to this construction of $X^{h \alpha}$ in Section 3.

For any space $X$, set $\widehat{H}^{i}\left(X ; \mathbb{Z}_{p}\right)=\lim H^{i}\left(X ; \mathbb{Z} / p^{k}\right)$ for each $i$, and let $\widehat{H}^{*}\left(X ; \mathbb{Z}_{p}\right)$ be the sum of the $\widehat{H}^{i}\left(X ; \mathbb{Z}_{p}\right)$. If $H^{*}\left(X ; \mathbb{F}_{p}\right)$ is finite in each degree, then $\widehat{H}^{*}\left(X ; \mathbb{Z}_{p}\right)$ is isomorphic to the usual cohomology ring $H^{*}\left(X ; \mathbb{Z}_{p}\right)$ with coefficients in the $p$ adics.

Recall that $\operatorname{Out}(X)$ denotes the group of homotopy classes of homotopy selfequivalences of a space $X$. By the $p$-adic topology on $\operatorname{Out}(X)$, we mean the topology for which $\left\{U_{k}\right\}$ is a basis of open neighborhoods of the identity, where $U_{k} \leq \operatorname{Out}(X)$ is the group of automorphisms which induce the identity on $H^{*}\left(X ; \mathbb{Z} / p^{k}\right)$. Thus this topology is Hausdorff if and only if $\operatorname{Out}(X)$ is detected on $\widehat{H}^{*}\left(X ; \mathbb{Z}_{p}\right)$.

Theorem 2.4. Fix a prime $p$. Let $X$ be a connected, $p$-complete space such that

- $H^{*}\left(X ; \mathbb{F}_{p}\right)$ is Noetherian, and

- $\operatorname{Out}(X)$ is detected on $\widehat{H}^{*}\left(X ; \mathbb{Z}_{p}\right)$.

Let $\alpha$ and $\beta$ be homotopy self-equivalences of $X$ which generate the same closed subgroup of $\operatorname{Out}(X)$ under the $p$-adic topology. Then $X^{h \alpha} \simeq X^{h \beta}$.

Proof. Upon replacing $X$ by the double mapping telescope of $\alpha$ (Remark 2.2), we can assume that $\alpha$ is a homeomorphism. By Lemma B.1, this does not change the homotopy type of $X^{h \alpha}$ or of $X^{h \beta}$.

Let $r \geq 1$ be the smallest integer prime to $p$ such that the action of $\alpha^{r}$ on $H^{*}\left(X ; \mathbb{F}_{p}\right)$ has $p$-power order. (The action of $\alpha$ on the Noetherian ring $H^{*}\left(X ; \mathbb{F}_{p}\right)$ has finite order.) Since $\overline{\langle\alpha\rangle}=\overline{\langle\beta\rangle}, H^{*}\left(\alpha ; \mathbb{F}_{p}\right)$ and $H^{*}\left(\beta ; \mathbb{F}_{p}\right)$ generate the same subgroup in $\operatorname{Aut}\left(H^{*}\left(X ; \mathbb{F}_{p}\right)\right)$, hence have the same order, and so $r$ is also the smallest integer prime to $p$ such that the action of $\beta^{r}$ on $H^{*}\left(X ; \mathbb{F}_{p}\right)$ has $p$-power order.

Let

$$
p_{\alpha}: X_{h \alpha} \longrightarrow S^{1} \quad \text { and } \quad p_{\beta}: X_{h \beta} \longrightarrow S^{1}
$$

be the canonical projections (Definition 2.1). Let

$$
\widetilde{p}_{\alpha}: \widetilde{X}_{h \alpha} \longrightarrow S^{1} \quad \text { and } \quad \tilde{p}_{\beta}: \tilde{X}_{h \beta} \longrightarrow S^{1}
$$

be their $r$-fold cyclic covers, where we take the $r$-fold cover of $S^{1}$ as well as of $X_{h \alpha}$ and $X_{h \beta}$. We regard $\widetilde{p}_{\alpha}$ and $\widetilde{p}_{\beta}$ as equivariant maps between spaces with $\mathbb{Z} / r$-action. Then

$$
\widetilde{X}_{h \alpha} \cong(X \times I \times(\mathbb{Z} / r)) / \sim \cong(X \times[0, r]) / \approx,
$$

where $(x, 0, i) \sim(\alpha(x), 1, i-1)$ and $(x, 0) \approx\left(\alpha^{r}(x), r\right)$. In other words, $\tilde{X}_{h \alpha} \simeq X_{h \alpha^{r}}$ (and $\widetilde{p}_{\alpha}$ is its canonical fibration), and $\widetilde{X}_{h \beta} \cong X_{h \beta^{r}}$. Also, there is a bijection between sections of the bundle $p_{\alpha}$ and $\mathbb{Z} / r$-equivariant sections of $\widetilde{p}_{\alpha}$ : each section of $p_{\alpha}$ is covered by a unique equivariant section of $\tilde{p}_{\alpha}$ (by covering space theory), and each equivariant section induces a section of $p_{\alpha}$ by taking the orbit map. Thus $X^{h \alpha}$ is the space of all equivariant sections of $\widetilde{p}_{\alpha}$. 
Since $\alpha^{r}$ acts on $H^{*}\left(X ; \mathbb{F}_{p}\right)$ with order a power of $p$, this action is nilpotent, and by [BK, II.5.1], the homotopy fiber of $\widetilde{p}_{\alpha p} \wedge$ has the homotopy type of $X_{p}^{\wedge} \simeq X$. Thus the rows in the following diagram are (homotopy) fibration sequences:

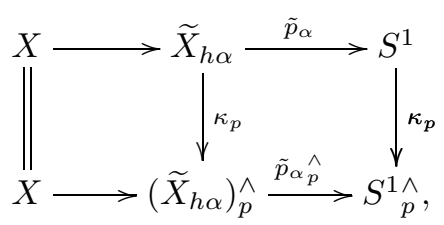

and so the right-hand square is a homotopy pullback. By the definition of $p$ completion in [BK], the induced actions of $\mathbb{Z} / r$ on $S_{p}^{1 \wedge}$ and on $\left(\widetilde{X}_{h \alpha}\right)_{p}^{\wedge}$ are free, since the actions on the uncompleted spaces are free. Hence $X^{h \alpha}$ can be described (up to homotopy), not only as the space of $\mathbb{Z} / r$-equivariant sections of $\widetilde{p}_{\alpha}$, but also as the space of $\mathbb{Z} / r$-equivariant liftings of $\kappa_{p}\left(S^{1}\right): S^{1} \longrightarrow S_{p}^{1 \wedge}$ along $\widetilde{p}_{\alpha p} \wedge$. In other words,

$$
X^{h \alpha} \simeq \operatorname{fiber}\left(\operatorname{map}_{\mathbb{Z} / r}\left(S^{1},\left(\widetilde{X}_{h \alpha}\right)_{p}^{\wedge}\right) \stackrel{\widetilde{p}_{\alpha \hat{p}^{\circ}-}}{\longrightarrow} \operatorname{map}_{\mathbb{Z} / r}\left(S^{1}, S_{p}^{1 \wedge}\right)\right)
$$

(the fiber over $\kappa_{p}\left(S_{1}\right)$ ).

Consider again the $p$-completed fibration sequence

$$
X \longrightarrow\left(\widetilde{X}_{h \alpha}\right)_{p}^{\wedge} \stackrel{\widetilde{p}_{\alpha}}{\longrightarrow} B S_{p}^{1 \wedge},
$$

and its orbit fibration

$$
X \longrightarrow\left(\widetilde{X}_{h \alpha}\right)_{p}^{\wedge} /(\mathbb{Z} / r) \stackrel{\widehat{p}_{\alpha}}{\longrightarrow} B S_{p}^{1 \wedge} /(\mathbb{Z} / r) .
$$

Here, $\pi_{1}\left(B S_{p}^{1 \wedge}\right) \cong \mathbb{Z}_{p}$, and $\pi_{1}\left(B S_{p}^{1 \wedge} /(\mathbb{Z} / r)\right) \cong \mathbb{Z}_{p} \times \mathbb{Z} / r$ (the completion of $\mathbb{Z}$ with respect to the ideals $\left.r p^{i} \mathbb{Z}\right)$. Since $\alpha^{r}$ acts on $H^{*}(X ; \mathbb{Z} / p)$ with order a power of $p$, it also acts on each $H^{*}\left(X ; \mathbb{Z} / p^{k}\right)$ with order a power of $p$, and hence the homotopy action of $\pi_{1}\left(B S_{p}^{1 \wedge}\right)$ on $X$ has as image the $p$-adic closure of $\left\langle\alpha^{r}\right\rangle$. Thus the homotopy action of $\pi_{1}\left(B S_{p}^{1 \wedge} /(\mathbb{Z} / r)\right)$ on $X$, defined by the fibration $\widehat{p}_{\alpha}$, has as image the $p$-adic closure of $\langle\alpha\rangle$.

Since $\beta \in \overline{\langle\alpha\rangle}$ by assumption, we can represent it by a map $S^{1} \stackrel{b}{\longrightarrow} S_{p}^{1 \wedge} /(\mathbb{Z} / r)$. Let $Y$ be the homotopy pullback defined by the following diagram:

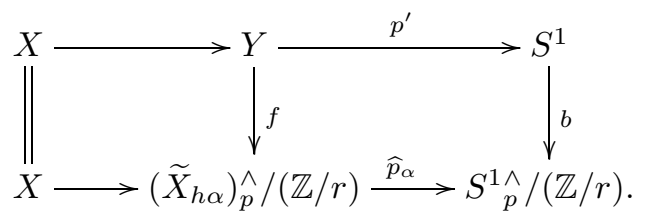

Thus the canonical generator of $\pi_{1}\left(S^{1}\right)$ induces $[\beta] \in \operatorname{Out}(X)$. By Remark 2.2, $p^{\prime}: Y \longrightarrow S^{1}$ is homeomorphic to $p_{\beta^{\prime}}: X_{h \beta^{\prime}} \longrightarrow S^{1}$ for some homeomorphism $\beta^{\prime}$ of $X$ which is homotopic to $\beta$. Upon taking $r$-fold covers and then completing the first row, this induces a map of fibrations

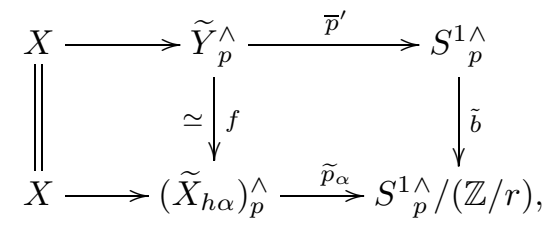


which is an equivalence since $\widetilde{b}$ is an equivalence (since $\langle\beta\rangle$ is dense in $\overline{\langle\alpha\rangle}$ ). Also, $\left(\widetilde{Y}_{p}^{\wedge}, \widetilde{p}^{\prime}\right) \cong\left(\left(\widetilde{X}_{h \beta^{\prime}}\right)_{p}^{\wedge}, \widetilde{p}_{\beta^{\prime}}\right)$, and $f$ and $\widetilde{b}$ are equivariant with respect to some automorphism of $\mathbb{Z} / r$.

The maps $S^{1} \longrightarrow S_{p}^{1 \wedge} \longleftarrow(X)_{h \mathbb{Z}_{p}}$ determine a commutative diagram

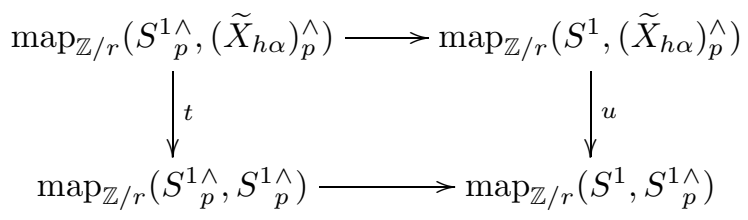

which in turn induces a map between the respective fibers. The horizontal arrows in (21) are homotopy equivalences because the target spaces in the respective mapping spaces are $p$-complete and $\mathbb{Z} / r$ acts freely on the source spaces. Hence the fibers of the vertical maps in (2) are homotopy equivalent. Since $u$ has fiber $X^{h \alpha}$ by (1), this proves that $X^{h \alpha}$ has the homotopy type of the space of equivariant sections of the bundle $\left(X_{h \alpha}\right)_{p}^{\wedge} \stackrel{\widetilde{p}_{\alpha \hat{p}}}{\longrightarrow} S_{p}^{1 \wedge}$. Since this bundle is equivariantly equivalent to the one with total space $\left(\widetilde{X}_{h \beta^{\prime}}\right)_{p}^{\wedge}$, the same argument applied to $\beta^{\prime}$ (together with Lemma B.1) proves that $X^{h \alpha} \simeq X^{h \beta}$.

Our main application of Theorem 2.4 is to the case where $X=B G_{p}^{\wedge}$ for a compact connected Lie group $G$.

Corollary 2.5. Let $G$ be a compact connected Lie group, and let $\alpha, \beta \in \operatorname{Out}\left(B G_{p}^{\wedge}\right)$ be two self-equivalences of the $p$-completed classifying space. If $\alpha$ and $\beta$ generate the same closed subgroup of $\operatorname{Out}\left(B G_{p}^{\wedge}\right)$, then $\left(B G_{p}^{\wedge}\right)^{h \alpha} \simeq\left(B G_{p}^{\wedge}\right)^{h \beta}$.

Proof. From the spectral sequence for the fibration $U(n) / G \longrightarrow B G \longrightarrow B U(n)$ for any embedding $G \leq U(n)$, we see that $H^{*}\left(B G ; \mathbb{F}_{p}\right)$ is Noetherian. By [JMO, Theorem 2.5], Out $\left(B G_{p}^{\wedge}\right)$ is detected by its restriction to $B T_{p}^{\wedge}$ for a maximal torus $T$, and hence by invariant theory is detected by $\mathbb{Q} \otimes_{\mathbb{Z}} \widehat{H}^{*}\left(B G ; \mathbb{Z}_{p}\right)$. So the hypotheses of Theorem 2.4 hold when $X=B G_{p}^{\wedge}$.

The hypotheses on $X$ in Theorem 2.4 also apply whenever $X$ is the classifying space of a connected $p$-compact group. The condition on cohomology holds by DW, Theorem 2.3]. Automorphisms are detected by restriction to the maximal torus by [AGMV, Theorem 1.1] (when $p$ is odd) and [Ml, Theorem 1.1] (when $p=2$ ).

\section{Finite groups of Lie type}

We first fix our terminology. Let $\mathbb{G}$ be a connected reductive group scheme over $\mathbb{Z}$. Thus for each algebraically closed field $K, \mathbb{G}(K)$ is a complex connected algebraic group such that for some finite central subgroup $Z \leq Z(\mathbb{G}(K)), \mathbb{G}(K) / Z$ is the product of a $K$-torus and a semisimple group. For any prime power $q$, we let $\mathbb{G}(q)$ be the fixed subgroup of the field automorphism $\psi^{q}$. Also, if $\tau$ is any automorphism of $\mathbb{G}$ of finite order, then ${ }^{\tau} \mathbb{G}(q)$ will denote the fixed subgroup of the composite $\tau \psi^{q}$ on $\mathbb{G}\left(\overline{\mathbb{F}}_{q}\right)$.

Note that with this definition, when $\mathbb{G}=P S L_{n}, \mathbb{G}(q)$ does not mean $P S L_{n}(q)$ in the usual sense, but rather its extension by diagonal automorphisms (i.e., $P G L_{n}(q)$ ). By Proposition 1.4, however, any equivalence between fusion systems over groups $S L_{n}(-)$ will also induce an equivalence between fusion systems over $P S L_{n}(-)$. 
Also, we are not including the case $\mathbb{G}=S O_{n}$ for $n$ even, since $S O_{n}\left(\overline{\mathbb{F}}_{2}\right)$ is not connected. Instead, when working with orthogonal groups in even dimensions, we take $\mathbb{G}=\Omega_{n}$ (and $\Omega_{n}(K)=S O_{n}(K)$ when $K$ is algebraically closed of characteristic different from two).

The results in this section are based on Corollary 2.5, together with the following theorem of Friedlander. Following the terminology of [GLS3], we define a Steinberg endomorphism of an algebraic group $\bar{G}$ over an algebraically closed field to be an algebraic endomorphism $\psi: \bar{G} \longrightarrow \bar{G}$ which is bijective, and whose fixed subgroup is finite. For any connected complex Lie group $\mathbb{G}(\mathbb{C})$ with maximal torus $\mathbb{T}(\mathbb{C})$, any prime $p$, and any $m \in \mathbb{Z}$ prime to $p, \Psi^{m}: B \mathbb{G}(\mathbb{C})_{p}^{\wedge} \longrightarrow B \mathbb{G}(\mathbb{C})_{p}^{\wedge}$ denotes a selfequivalence whose restriction to $B \mathbb{T}(\mathbb{C})_{p}^{\wedge}$ is induced by $\left(x \mapsto x^{m}\right)$ (an "unstable Adams operation"). Such a map is unique up to homotopy by [JMO, Theorem 2.5] (applied to $B G \simeq B \mathbb{G}(\mathbb{C})$, where $G$ is a maximal compact subgroup of $\mathbb{G}(\mathbb{C})$ ).

Theorem 3.1. Fix a connected reductive group scheme $\mathbb{G}$ over $\mathbb{Z}$, a prime power $q$, and a prime $p$ which does not divide $q$. Then for any Steinberg endomorphism $\psi$ of $\mathbb{G}\left(\overline{\mathbb{F}}_{q}\right)$ with fixed subgroup $H$,

$$
B H_{p}^{\wedge} \simeq\left(B \mathbb{G}(\mathbb{C})_{p}^{\wedge}\right)^{h \Psi}
$$

for some $\Psi: B \mathbb{G}(\mathbb{C})_{p}^{\wedge} \stackrel{\simeq}{\longrightarrow} B \mathbb{G}(\mathbb{C})_{p}^{\wedge}$. If $\psi=\tau\left(\overline{\mathbb{F}}_{q}\right) \circ \mathbb{G}\left(\psi^{q}\right)$, where $\tau \in \operatorname{Aut}(\mathbb{G})$ and $\psi^{q} \in \operatorname{Aut}\left(\overline{\mathbb{F}}_{q}\right)$ is the automorphism $\left(x \mapsto x^{q}\right)$, then $\Psi \simeq B \tau(\mathbb{C}) \circ \Psi^{q}$, where $\Psi^{q}$ is as described above.

Proof. By [Fr, Theorem 12.2], there is a commutative square

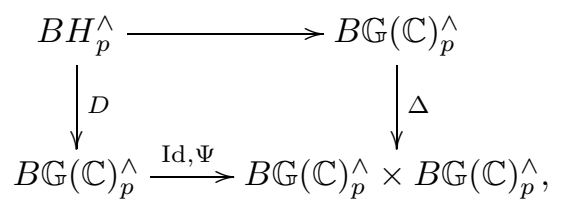

where $\Delta$ is the diagonal map, and where the induced map $\operatorname{fib}(D) \stackrel{f}{\longrightarrow} \operatorname{fib}(\Delta)$ between the homotopy fibers induces an isomorphism in $\bmod p$ cohomology. Since $B \mathbb{G}(\mathbb{C})$ is simply connected, $\operatorname{fib}(D)$ and $\mathrm{fib}(\Delta)$ are both $p$-complete by the Mod- $R$ fibre lemma of Bousfield and Kan [BK, II.5.1]. Hence $f$ is a homotopy equivalence by [BK, I.5.5], and so the above square is a homotopy pullback. Thus $B H_{p}^{\wedge} \simeq$ $\left(B \mathbb{G}(\mathbb{C})_{p}^{\wedge}\right)^{h \Psi}$ by Remark 2.3 .

From the proof of Friedlander's theorem, one sees that $\Psi$ is induced by $B \psi$, together with the homotopy equivalence $B \mathbb{G}(\mathbb{C})_{p}^{\wedge} \simeq \operatorname{holim}\left(\left(B \mathbb{G}\left(\overline{\mathbb{F}}_{q}\right)_{\text {et }}\right)_{p}^{\wedge}\right)$ of $[\mathrm{Fr}$, Proposition 8.8]. This equivalence is natural with respect to the inclusion of a maximal torus $\mathbb{T}$ in $\mathbb{G}$. Hence when $\psi=\tau\left(\overline{\mathbb{F}}_{q}\right) \circ \mathbb{G}\left(\psi^{q}\right), \Psi$ restricts to the action on $B \mathbb{T}(\mathbb{C})_{p}^{\wedge}$ induced by $\tau$ and $\left(x \mapsto x^{q}\right)$.

Theorem 3.1 can now be combined with Corollary 2.5 to prove Theorem A, i.e., to compare fusion systems over different Chevalley groups associated to the same connected group scheme $\mathbb{G}$. This will be done in the next two propositions.

Proposition 3.2. Fix a prime $p$, a connected reductive group scheme $\mathbb{G}$ over $\mathbb{Z}$, and an automorphism $\tau$ of $\mathbb{G}$ of finite order $k$. Assume, for each $m$ prime to $k$, that $\tau^{m}$ is conjugate to $\tau$ in the group of all automorphisms of $\mathbb{G}$. Let $q$ and $q^{\prime}$ be 
prime powers prime to $p$. Assume either

(a) $\overline{\langle q\rangle}=\overline{\left\langle q^{\prime}\right\rangle}$ as subgroups of $\mathbb{Z}_{p}^{\times}$, or

(b) there is some $\psi^{-1}$ in the Weyl group of $\mathbb{G}$ which inverts all elements of the maximal torus, and $\overline{\langle-1, q\rangle}=\overline{\left\langle-1, q^{\prime}\right\rangle}$ as subgroups of $\mathbb{Z}_{p}^{\times}$.

Then there is an isotypical equivalence $\mathcal{F}_{p}\left({ }^{\tau} \mathbb{G}(q)\right) \simeq \mathcal{F}_{p}\left({ }^{\tau} \mathbb{G}\left(q^{\prime}\right)\right)$.

Proof. Since $H^{*}\left(B \mathbb{G}(\mathbb{C})_{p}^{\wedge} ; \mathbb{F}_{p}\right)$ is Noetherian and finite in each degree, each of the rings $H^{*}\left(B \mathbb{G}(\mathbb{C})_{p}^{\wedge} ; \mathbb{Z} / p^{k}\right)$ has a finite automorphism group. So if $\overline{\langle q\rangle}=\overline{\left\langle q^{\prime}\right\rangle}$, then $\Psi^{q}$ and $\Psi^{q^{\prime}}$ generate the same subgroup in $\operatorname{Aut}\left(H^{*}\left(B \mathbb{G}(\mathbb{C})_{p}^{\wedge} ; \mathbb{Z} / p^{k}\right)\right)$ for each $k$, and hence the same closed subgroup in $\operatorname{Out}\left(B \mathbb{G}(\mathbb{C})_{p}^{\wedge}\right)$.

Since $\tau$ is an automorphism of $\mathbb{G}$, its actions on $\mathbb{G}\left(\overline{\mathbb{F}}_{q}\right)$ and $\mathbb{G}\left(\overline{\mathbb{F}}_{q^{\prime}}\right)$ commute with the field automorphisms $\psi^{q}$ and $\psi^{q^{\prime}}$. Thus $\left(\tau \psi^{q}\right)^{k}=\psi^{q^{k}}$ has a finite fixed subgroup, so $\tau \psi^{q}$ also has a finite fixed subgroup, and similarly for $\tau \psi^{q^{\prime}}$. So by Theorem 3.1

$$
B\left({ }^{\tau} \mathbb{G}(q)\right)_{p}^{\wedge} \simeq\left(B \mathbb{G}(\mathbb{C})_{p}^{\wedge}\right)^{h\left(B \tau \circ \Psi^{q}\right)} \quad \text { and } \quad B\left({ }^{\tau} \mathbb{G}\left(q^{\prime}\right)\right)_{p}^{\wedge} \simeq\left(B \mathbb{G}(\mathbb{C})_{p}^{\wedge}\right)^{h\left(B \tau \circ \Psi^{q^{\prime}}\right.} .
$$

Assume $\overline{\langle q\rangle}=\overline{\left\langle q^{\prime}\right\rangle}$. Then for some $m$ prime to $k=|\tau|, q \equiv\left(q^{\prime}\right)^{m}$ modulo $\overline{\left\langle q^{k}\right\rangle}=\overline{\left\langle q^{\prime k}\right\rangle}$. Hence $B \tau^{m} \circ \Psi^{q}$ and $B \tau \circ \Psi^{q^{\prime}}$ generate the same closed subgroup of Out $\left(\mathbb{G}(\mathbb{C})_{p}^{\wedge}\right)$ under the $p$-adic topology, since they generate the same subgroup modulo $\overline{\left\langle\Psi^{q^{k}}\right\rangle}$. Thus

$$
\left(B \mathbb{G}(\mathbb{C})_{p}^{\wedge}\right)^{h\left(B \tau \circ \Psi^{q^{\prime}}\right)} \simeq\left(B \mathbb{G}(\mathbb{C})_{p}^{\wedge}\right)^{h\left(B \tau^{m} \circ \Psi^{q}\right)} \simeq\left(B \mathbb{G}(\mathbb{C})_{p}^{\wedge}\right)^{h\left(B \tau \circ \Psi^{q}\right)},
$$

where the first equivalence holds by Corollary 2.5] and the second since $\tau$ and $\tau^{m}$ are conjugate in the group of all automorphisms of $\mathbb{G}$. So $B\left({ }^{\tau} \mathbb{G}(q)\right)_{p}^{\wedge} \simeq B\left({ }^{\tau} \mathbb{G}\left(q^{\prime}\right)\right)_{p}^{\wedge}$, and there is an isotypical equivalence between the fusion systems of these groups by Theorem 1.5 .

If - Id is in the Weyl group, then by JMO, Theorem 2.5], there is a homomorphism $\mathbb{Z}_{p}^{\times} /\{ \pm 1\} \longrightarrow \operatorname{Out}\left(B \mathbb{G}(\mathbb{C})_{p}^{\wedge}\right)$ which sends the class of $q$ to that of $\Psi^{q}$. So if $\overline{\langle-1, q\rangle}=\overline{\left\langle-1, q^{\prime}\right\rangle}$, then by the same argument as that just given, $\overline{\left\langle\Psi^{q}\right\rangle}=\overline{\left\langle\Psi^{q^{\prime}}\right\rangle}$, $\left(B \mathbb{G}(\mathbb{C})_{p}^{\wedge}\right)^{h\left(B \tau \circ \Psi^{q}\right)} \simeq\left(B \mathbb{G}(\mathbb{C})_{p}^{\wedge}\right)^{h\left(B \tau \circ \Psi^{q^{\prime}}\right)}$, and hence $\mathcal{F}_{p}(\tau \mathbb{G}(q)) \simeq \mathcal{F}_{p}\left(\tau \mathbb{G}\left(q^{\prime}\right)\right)$.

To make the condition $\overline{\langle q\rangle}=\overline{\left\langle q^{\prime}\right\rangle}$ more concrete, note that for any prime $p$, and any $q, q^{\prime}$ prime to $p$ of order $s$ and $s^{\prime}$, respectively, in $\mathbb{F}_{p}^{\times}$,

$$
\overline{\langle q\rangle}=\overline{\left\langle q^{\prime}\right\rangle} \Longleftrightarrow \begin{cases}s=s^{\prime} \text { and } v_{p}\left(q^{s}-1\right)=v_{p}\left(q^{\prime}-1\right) & \text { if } p \text { is odd, } \\ q \equiv q^{\prime}(\bmod 8) \text { and } v_{p}\left(q^{2}-1\right)=v_{p}\left(q^{\prime 2}-1\right) & \text { if } p=2 .\end{cases}
$$

When $p$ is odd, this follows from the decomposition

$$
\mathbb{Z}_{p}^{\times}=\mathbb{F}_{p}^{\times} \times\left(1+p \mathbb{Z}_{p}\right) \cong(\mathbb{Z} /(p-1)) \times \mathbb{Z}_{p}
$$

Each subgroup of $(\mathbb{Z} /(p-1)) \times\left(\mathbb{Z} / p^{n}\right)$ factors as a product of subgroups of the two factors, and hence each closed subgroup $H \leq \mathbb{Z}_{p}^{\times}$factors as a product $(\mathbb{Z} / s) \times(1+$ $p^{k} \mathbb{Z}_{p}$ ) for some $s \mid p-1$ and some $k \geq 1$. When $H=\overline{\langle q\rangle}$ as above, $s$ is the order of $q$ in $\mathbb{F}_{p}^{\times}$and $k=v_{p}\left(q^{s}-1\right)$. This proves the above claim when $p$ is odd, and that when $p=2$ follows by a similar argument using the decomposition $\mathbb{Z}_{2}^{\times}=\{ \pm 1\} \times\left(1+4 \mathbb{Z}_{2}\right)$. 
Corollary 2.5 can also be applied to compare fusion systems of Steinberg groups with those of related Chevalley groups.

Proposition 3.3. Fix a prime $p$, and a pair $q, q^{\prime}$ of prime powers prime to $p$ such that $\overline{\langle-q\rangle}=\overline{\left\langle q^{\prime}\right\rangle}$ as subgroups of $\mathbb{Z}_{p}^{\times}$. Assume $\mathbb{G}$ is of type $A_{n}, D_{n}$ for $n$ odd, or $E_{6}$, and $\tau$ is a graph automorphism of $\mathbb{G}$ of order two. Then $\mathcal{F}_{p}\left({ }^{\tau} \mathbb{G}(q)\right)$ and $\mathcal{F}_{p}\left(\mathbb{G}\left(q^{\prime}\right)\right)$ are isotypically equivalent. In other words, there are isotypical equivalences:

(a) $\mathcal{F}_{p}\left(S U_{n}(q)\right) \simeq \mathcal{F}_{p}\left(S L_{n}\left(q^{\prime}\right)\right)$ for all $n$.

(b) $\mathcal{F}_{p}\left(\operatorname{Spin}_{2 n}^{-}(q)\right) \simeq \mathcal{F}_{p}\left(\operatorname{Spin}_{2 n}^{+}\left(q^{\prime}\right)\right)$ for all odd $n$.

(c) $\mathcal{F}_{p}\left({ }^{2} E_{6}(q)\right) \simeq \mathcal{F}_{p}\left(E_{6}\left(q^{\prime}\right)\right)$.

Proof. Set $\mathbb{G}=S L_{n}, \operatorname{Spin}_{2 n}$ for $n$ odd, or the simply connected $E_{6}$; and let $\tau$ be the graph automorphism of order two. In all of these cases, $\tau$ acts by inverting the elements of some maximal torus. Hence by Theorem 3.1, $B\left({ }^{\tau} \mathbb{G}(q)\right)_{p}^{\wedge} \simeq\left(B \mathbb{G}(\mathbb{C})_{p}^{\wedge}\right)^{h \Psi^{-q}}$ and $B \mathbb{G}\left(q^{\prime}\right)_{p}^{\wedge} \simeq\left(B \mathbb{G}_{p}^{\wedge}\right)^{h \Psi^{q^{\prime}}}$. So $B \mathbb{G}(q)_{p}^{\wedge} \simeq B \mathbb{G}\left(q^{\prime}\right)_{p}^{\wedge}$ by Corollary 2.5, [JMO, Theorem 2.5], and the assumption $\overline{\langle-q\rangle}=\overline{\left\langle q^{\prime}\right\rangle}$, and there is an isotypical equivalence between the fusion systems of these groups by Theorem 1.5 .

Upon combining this with Proposition 1.4, one gets similar results for $P S U_{n}(q)$, $\Omega_{2 n}^{-}(q), P \Omega_{2 n}^{-}(q)$, etc.

\section{More homotopy FiXed POINTS}

To get more results of this type, we need to look at homotopy fixed points in a more general setting. Let $G$ be any discrete group, and let $E G$ be a universal space for $G$, i.e., a contractible $C W$-complex on which $G$ acts by freely permuting the cells. Thus $B G=E G / G$ is a classifying space for $G$. If $G$ acts on a space $X$, then the homotopy fixed point space for the action is the $\operatorname{space} X^{h G} \stackrel{\text { def }}{=} \operatorname{map}_{G}(E G, X)$. The usual fixed point space $X^{G}$ can be identified as the subspace of all constant maps in $X^{h G}$.

Remark 4.1. Unlike the usual fixed point space, $(-)^{h G}$ has the property that if $f: X \stackrel{\simeq}{\longrightarrow} Y$ is $G$-equivariant and a homotopy equivalence, then it induces a homotopy equivalence $f^{h G}: X^{h G} \stackrel{\simeq}{\longrightarrow} Y^{h G}$. Formally, this follows as a special case of the homotopy invariance of homotopy inverse limits (see [BK, XI.5.6]). But it is in fact much more elementary: in the following diagram, one can always find a $G$-equivariant lifting

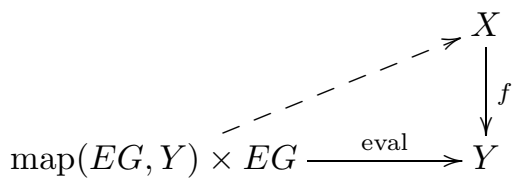

since $f$ is a homotopy equivalence and $G$ acts freely on the source space.

When $G=\mathbb{Z}$, we can take $E \mathbb{Z}=\mathbb{R}$, where $\mathbb{Z}$ acts by translation. If $\mathbb{Z}$ acts on $X$, where $1 \in \mathbb{Z}$ acts via a homeomorphism $\alpha$, then $X^{h \mathbb{Z}}=\operatorname{map}_{\mathbb{Z}}(\mathbb{R}, X)$ is homeomorphic to the space of maps $\phi:[0,1] \longrightarrow X$ such that $\phi(1)=\alpha(\phi(0))$, i.e. to $X^{h \alpha}$. So the homotopy fixed set of a homeomorphism is just a special case of the homotopy fixed set of a group action. 
Theorem 4.2. Fix a prime $p$. Let $X$ be a connected, $p$-complete space such that - $H^{*}\left(X ; \mathbb{F}_{p}\right)$ is Noetherian, and

- $\operatorname{Out}(X)$ is detected on $\widehat{H}^{*}\left(X ; \mathbb{Z}_{p}\right)$.

Fix a finite cyclic group $K=\langle g\rangle$ of order $r$ prime to $p$, together with an action of $K$ on $X$. Let $\alpha: X \longrightarrow X$ be a homotopy self-equivalence of $X$ and assume it extends to a $K$-equivariant map

$$
\beta: E K \times X \stackrel{\simeq}{\longrightarrow} X .
$$

Assume $H^{*}\left(\alpha ; \mathbb{F}_{p}\right)$ is an automorphism of $H^{*}\left(X ; \mathbb{F}_{p}\right)$ of p-power order (equivalently, the action is nilpotent). Let $\kappa: X \longrightarrow X$ be the action of a generator $g \in K$. Then

$$
X^{h(\kappa \alpha)} \simeq\left(X^{h K}\right)^{h \alpha} .
$$

Proof. Upon replacing $X$ by the double mapping telescope of $\operatorname{pr}_{E K} \times \beta$ (Remark 2.2), and using Remark 4.1. we can assume that $X$ has a free action of the group $K \times \mathbb{Z}$, and hence that $\kappa$ and $\alpha$ are commuting homeomorphisms of $X$ which are the actions of generators of $K$ and of $\mathbb{Z}$. In particular, $(\kappa \alpha)^{r}=\alpha^{r}$.

For each $k \geq 1, \alpha$ acts on $H^{*}\left(X ; \mathbb{Z} / p^{k}\right)$ as an automorphism of $p$-power order. Since $r$ is prime to $p$, this implies that $\alpha$ and $\alpha^{r}$ generate the same closed subgroup of $\operatorname{Out}(X)$. So by Theorem [2.4 the inclusion of $X^{h \alpha}$ into $X^{h \alpha^{r}}$ is a homotopy equivalence.

The theorem will follow once we check the following equivalences:

$$
\begin{aligned}
\left(X^{h K}\right)^{h \alpha} & =\operatorname{map}_{\alpha}\left(\mathbb{R}, \operatorname{map}_{K}(E K, X)\right) \cong \operatorname{map}_{K}\left(E K, \operatorname{map}_{\alpha}(\mathbb{R}, X)\right) \\
& \simeq \operatorname{map}_{\alpha \kappa}\left(E K, \operatorname{map}_{\alpha^{r}}(\mathbb{R}, X)\right) \cong \operatorname{map}_{\alpha \kappa}(E K \times \mathbb{R}, X) \simeq X^{h \alpha \kappa} .
\end{aligned}
$$

Here, $\alpha$ acts on $\mathbb{R}$ by translation (and $K$ acts trivially). The two homeomorphisms

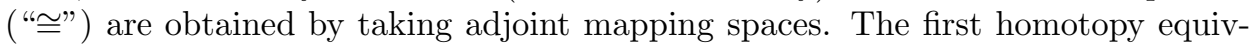
alence holds since the inclusion of $\operatorname{map}_{\alpha}(\mathbb{R}, X)=X^{h \alpha}$ into $\operatorname{map}_{\alpha^{r}}(\mathbb{R}, X)=X^{h \alpha^{r}}$ is $K$-equivariant and a homotopy equivalence, where the $K$-action on the second space is induced by the actions of $\alpha \kappa$ on $\mathbb{R}$ and on $X$ (note that $(\alpha \kappa)^{r}=\alpha^{r}$ acts trivially). The second holds since the inclusion of $\mathbb{R}$ into $E K \times \mathbb{R}$ (induced by the inclusion of $\langle\alpha \kappa\rangle \cong \mathbb{Z}$ into $K \times\langle\alpha\rangle$ ) is an equivariant homotopy equivalence (both spaces are contractible with free action of $\langle\alpha \kappa\rangle \cong \mathbb{Z}$ ).

The following more technical lemma will be needed when applying Theorem 4.2,

Lemma 4.3. Let $G$ be a compact connected semisimple Lie group, and let $K \leq$ $\operatorname{Aut}(G)$ be a finite group of automorphisms. If $p$ does not divide the order of $K$, then for any $q \in\left(\mathbb{Z}_{p}\right)^{\times}$, and any unstable Adams map $\Psi^{q}$, there is a $K$-equivariant map

$$
E K \times B G_{p}^{\wedge} \stackrel{f}{\longrightarrow} B G_{p}^{\wedge}
$$

whose restriction to $* \times B G_{p}^{\wedge}$ is $\Psi^{q}$.

Proof. The action of $K$ on $G$ induces an action of $K$ on the $p$-completed classifying space $B G_{p}^{\wedge}$. We will construct a $K$-equivariant map

$$
f_{0}: E K \longrightarrow \operatorname{map}\left(B G_{p}^{\wedge}, B G_{p}^{\wedge}\right)
$$

with image in the connected component of $\Psi^{q}$. By [JMO, Proposition 2.7], this component has the homotopy type of $B Z(G)_{p}^{\wedge} \simeq B Z(G)_{(p)}$, where $Z(G)_{(p)}$ denotes the $p$-power torsion in $Z(G)$. By equivariant obstruction theory $\mathrm{Br}$, Theorem 
II.1.5], the only obstruction to the existence of such a map lies in $H_{K}^{2}\left(E K ; Z(G)_{(p)}\right)$ (the obstruction to defining $f_{0}$ on 2-cells in $\left.E K\right)$. Also, $H_{K}^{2}\left(E K ; Z(G)_{(p)}\right) \cong$ $H^{2}\left(K ; Z(G)_{(p)}\right)$ by $[\mathrm{Br}$. I.9.3], and this group vanishes since $|K|$ is prime to $p$.

Thus there is a map $f_{0}$ as above, which is adjoint to the required equivariant map $f: E K \times B G_{p}^{\wedge} \longrightarrow B G_{p}^{\wedge}$.

The following result is one example of how Theorem 4.2 can be applied, in order to compare the fusion systems of $G_{2}(q)$ and ${ }^{3} D_{4}(q)$, and those of $F_{4}(q)$ and ${ }^{2} E_{6}(q)$. The first case was suggested by the lists of maximal subgroups of these groups in K11 and [K12, and also by the cohomology calculations in [FM] and Mi].

Example 4.4. Fix a prime $p$, and a prime power $q \equiv 1(\bmod p)$. Then the following hold.

(a) If $p \neq 3$, the fusion systems $\mathcal{F}_{p}\left(G_{2}(q)\right)$ and $\mathcal{F}_{p}\left({ }^{3} D_{4}(q)\right)$ are isotypically equivalent.

(b) If $p \neq 2$, the fusion systems $\mathcal{F}_{p}\left(F_{4}(q)\right)$ and $\mathcal{F}_{p}\left({ }^{2} E_{6}(q)\right)$ are isotypically equivalent.

Proof. To prove (a), we apply Theorem 4.2, with $X=B \operatorname{Spin}_{8}(\mathbb{C})_{p}^{\wedge} \simeq B \operatorname{Spin}(8)_{p}^{\wedge}$, with $K \cong C_{3}$ having the action on $X$ induced by the triality automorphism, with $\alpha=\Psi^{q}$, and with $\beta: E K \times X \longrightarrow X$ a $K$-equivariant extension of $\alpha$ as constructed in Lemma 4.3 .

We first show that the inclusion of $G_{2}$ into $\operatorname{Spin}(8)$ induces a homotopy equivalence $\left(B G_{2}\right)_{p}^{\wedge} \simeq X^{h K}$. Since there is always a map from the fixed point set of an action to its homotopy fixed point set, the inclusion of $G_{2}(\mathbb{C}) \cong \operatorname{Spin}_{8}(\mathbb{C})^{K}$ (cf. GLS3, Theorem 1.15.2]) into $\operatorname{Spin}_{8}(\mathbb{C})$ induces maps $\left(B G_{2}\right)_{p}^{\wedge} \longrightarrow X^{h K} \longrightarrow X$. The first map is a monomorphism in the sense of Dwyer and Wilkerson [DW, §3.2], since the composite is a monomorphism.

By [BM, Theorem $\mathrm{B}(2)], X^{h K}$ is the classifying space of a connected $p$-compact group in the sense of Dwyer and Wilkerson [DW]. Hence by [BM, Theorem B(2)], $H^{*}\left(X^{h K} ; \mathbb{Q}_{p}\right)$ is the polynomial algebra generated by the coinvariants $Q H^{*}\left(X ; \mathbb{Q}_{p}\right)_{K}$, i.e., the coinvariants of the $K$-action on the polynomial generators of $H^{*}\left(\operatorname{Spin}_{8}(\mathbb{C}) ; \mathbb{Q}_{p}\right)$. For any compact connected Lie group $G$ with maximal torus $T$, $H^{*}(B G ; \mathbb{Q})$ is the ring of invariants of the action of the Weyl group on $H^{*}(B T ; \mathbb{Q})$ Bor, Proposition 27.1] and is a polynomial algebra with degrees listed in [ST, Table VII]. In particular, $H^{*}\left(X ; \mathbb{Q}_{p}\right)$ has polynomial generators in degrees $4,8,12,8$, and an explicit computation shows that $K$ fixes generators in degrees 4 and 12 . Thus $H^{*}\left(X^{h K} ; \mathbb{Q}_{p}\right) \cong H^{*}\left(B G_{2}(\mathbb{C}) ; \mathbb{Q}_{p}\right)$ (as graded $\mathbb{Q}_{p}$-algebras). It follows from [MN] Proposition 3.7] that $\left(B G_{2}\right)_{p}^{\wedge} \longrightarrow X^{h K}$ is an isomorphism of connected $p$-compact groups because it is a monomorphism and a rational isomorphism.

Since $H^{*}\left(X ; \mathbb{F}_{p}\right)$ is Noetherian and finite in each degree, its automorphism group is finite. Hence the group of all $r \in \mathbb{Z}_{p}^{\times}$such that $\Psi^{r}$ induces the identity on $H^{*}\left(X ; \mathbb{F}_{p}\right)$ has finite index and contains $1+p^{k} \mathbb{Z}_{p}$ for some $k \geq 1$. Since $q \equiv 1(\bmod$ $p$ ), the action of $\alpha=\Psi^{q}$ on $H^{*}\left(X ; \mathbb{F}_{p}\right)$ has order dividing $p^{k-1}$.

Now let $\kappa \in \operatorname{Aut}(X)$ generate the action of $K$. By Theorem 3.1

$$
X^{h(\kappa \alpha)} \simeq B\left({ }^{3} D_{4}(q)\right)_{p}^{\wedge} \quad \text { and } \quad\left(X^{h K}\right)^{h \alpha} \simeq\left(B G_{2 p} \wedge\right)^{h \alpha} \simeq B G_{2}(q)_{p}^{\wedge} .
$$

Hence $B\left({ }^{3} D_{4}(q)\right)_{p}^{\wedge} \simeq B G_{2}(q)_{p}^{\wedge}$ by Theorem 4.2 , and so these groups have isotypically equivalent $p$-fusion systems by Theorem 1.5 . This proves (a). 
Now set $X=B E_{6}(\mathbb{C})_{p}^{\wedge}$ and $K=\langle\tau\rangle$, where $\tau$ is an outer automorphism of order two. For each $k \geq 0, \tau$ acts on $H^{2 k}\left(X ; \mathbb{Q}_{p}\right)$ via $(-1)^{k}$ : this follows since $H^{*}\left(X ; \mathbb{Q}_{p}\right)$ injects into the cohomology of any maximal torus and $\tau$ acts on an appropriate choice of maximal torus via $\left(g \mapsto g^{-1}\right)$. Since $H^{*}\left(X ; \mathbb{Q}_{p}\right)$ is polynomial with generators in degrees $4,10,12,16,18,24$, $\mathrm{BM}$, Theorem $\mathrm{B}(2)]$ implies that $H^{*}\left(X^{h K} ; \mathbb{Q}_{p}\right)$ is polynomial with generators in degrees $4,12,16,24$, and hence is isomorphic to $H^{*}\left(B F_{4}(\mathbb{C}) ; \mathbb{Q}_{p}\right)$. The rest of the proof of $(\mathrm{b})$ is identical to that of (a).

By applying Theorem 4.2 to the graph automorphisms of classical groups and using similar arguments, we get additional equivalences $\mathcal{F}_{p}\left(S U_{2 n}(q)\right) \simeq \mathcal{F}_{p}\left(S p_{2 n}(q)\right)$, $\mathcal{F}_{p}\left(S U_{2 n+1}(q)\right) \simeq \mathcal{F}_{p}\left(\operatorname{Spin}_{2 n+1}(q)\right)$, and $\mathcal{F}_{p}\left(\operatorname{Spin}_{2 n}^{-}(q)\right) \simeq \mathcal{F}_{p}\left(\operatorname{Spin}_{2 n-1}(q)\right)$. In all cases, this holds for $p$ odd and for $q \equiv 1(\bmod p)$. However, more general relations of these types also follow from Proposition A.3 and Theorem $\mathrm{A}$

\section{ApPendix A. Examples FOR ClASSiCAL groups}

We prove here a few other cases of isotypical equivalences between fusion systems of classical groups. All of these are shown by much more elementary methods than those used in Sections 3 and 4.

Recall that a bilinear form $\mathfrak{b}$ on a $k$-vector field $V$ is symplectic if $\mathfrak{b}(v, v)=0$ for each $v \in V$. When $\operatorname{char}(k) \neq 2$, this is equivalent to $\mathfrak{b}$ being antisymmetric.

Lemma A.1. Fix a finite field $k$, and a finite group $G$ of odd order prime to $\operatorname{char}(k)$. Let $V$ be a finite-dimensional $k G$-module such that $V^{G}=0$ ( $V$ has trivial fixed component) and $V \cong V^{*}$.

(a) There are nondegenerate $G$-invariant quadratic and symplectic forms on $V$.

(b) If $\mathfrak{b}, \mathfrak{b}^{\prime}: V \times V \longrightarrow k$ are two nondegenerate $G$-invariant symplectic forms, then there is a $\beta \in \operatorname{Aut}_{k G}(V)$ such that $\mathfrak{b}^{\prime}(v, w)=\mathfrak{b}(\beta(v), \beta(w))$ for all $v, w \in$ $V$. If $\mathfrak{q}, \mathfrak{q}^{\prime}: V \longrightarrow k$ are two nondegenerate $G$-invariant quadratic forms, then there is $\beta \in \operatorname{Aut}_{k G}(V)$ such that $\mathfrak{q}^{\prime}=\mathfrak{q} \circ \beta$.

Proof. Write $V=V_{1} \oplus V_{2}$, where $V_{1}$ is generated by all nontrivial self-dual irreducible submodules of $V$, and $V_{2}$ is generated by all irreducible submodules which are not self-dual. Since $V$ and $V_{1}$ are self-dual, so is $V_{2}$. Any nondegenerate $G$ invariant bilinear or quadratic form on $V$ splits as an orthogonal direct sum of nondegenerate forms on $V_{1}$ and $V_{2}$, so it suffices to prove the lemma when $V=V_{1}$ or $V=V_{2}$.

For each $k G$-module $W$, let $\operatorname{Sym}_{G}^{+}(W)$ and $\operatorname{Sym}_{G}^{-}(W)$ be the sets of nondegenerate $G$-invariant symmetric and antisymmetric forms on $W$. We show in Steps 1 and 2 that $\operatorname{Sym}_{G}^{ \pm}\left(V_{i}\right) \neq \varnothing$ for $i=1,2$, and that all forms in each set are isomorphic to each other. This proves the lemma when $\operatorname{char}(k) \neq 2$.

If $\operatorname{char}(k)=2$, then the lemma follows from the results on symmetric bilinear forms, together with the following observations.

- Each $\mathfrak{b} \in \operatorname{Sym}_{G}^{ \pm}(V)$ is symplectic. To see this, note that since $\mathfrak{b}$ is symmetric and $V^{G}=0$, the function $v \mapsto \mathfrak{b}(v, v)^{1 / 2}$ lies in $\operatorname{Hom}_{k G}(V, k)=0$.

- There is a unique $G$-invariant quadratic form $\mathfrak{q}: V \longrightarrow k$ associated to each $\mathfrak{b} \in \operatorname{Sym}_{G}^{ \pm}(V)$. To see that there is at least one $\mathfrak{q}$, let $\mathfrak{q}_{0}$ be any (non$G$-invariant) quadratic form associated to $\mathfrak{b}$, and set $\mathfrak{q}(v)=\sum_{g \in G} \mathfrak{q}_{0}(g v)$. 
Then $\mathfrak{q}$ is $G$-invariant, and is a quadratic form associated to $\mathfrak{b}$ since $\mathfrak{b}$ is $G$-invariant, $|G|$ is odd, and $\operatorname{char}(k)=2$. If $\mathfrak{q}^{\prime}$ is another $G$-invariant quadratic form associated to $\mathfrak{b}$, then the function $v \mapsto\left(\mathfrak{q}^{\prime}(v)-\mathfrak{q}(v)\right)^{1 / 2}$ lies in $\operatorname{Hom}_{k G}(V, k)=0$, so $\mathfrak{q}^{\prime}=\mathfrak{q}$.

Step 1: Assume first that $V$ is a nontrivial irreducible $k G$-module such that $V \cong V^{*}$. Any nonzero $G$-invariant form $\mathfrak{b}: V \times V \longrightarrow k$ is nondegenerate (since $V$ is irreducible), and there exist such forms since $V \cong V^{*}$. If $\mathfrak{b} \neq 0$, then either $\mathfrak{b} \in \operatorname{Sym}_{G}^{+}(V)$ or $\mathfrak{b}-\mathfrak{b}^{t} \in \operatorname{Sym}_{G}^{-}(V)$ (where $\mathfrak{b}^{t}(v, w)=\mathfrak{b}(w, v)$ ). Thus at least one of these sets is nonempty.

Let $\bar{k}$ be the algebraic closure of $k$. Set $K=\operatorname{End}_{k G}(V)$ : a finite field extension of $k$. We can regard $V$ as an (irreducible) $K G$-module, and then $\bar{V} \stackrel{\text { def }}{=} \bar{k} \otimes_{K} V$ is irreducible as a $\bar{k} G$-module (cf. [A, 25.8]). Let $\chi_{\bar{V}}: G \longrightarrow \mathbb{C}$ be the character of $\bar{V}$ under some identification of $\bar{k}^{\times}$with a group of roots of unity in $\mathbb{C}$. (See [Se, $\S 18]$.$) If \bar{V} \cong \bar{V}^{*}$, then $\sum_{g \in G} \chi_{\bar{V}}\left(g^{2}\right) \neq 0$ : this is shown in BtD, Proposition II.6.8] for complex representations, and the same proof applies in this situation. Since $|G|$ is odd, each element of $G$ is the square of a unique element, and hence $\sum_{g \in G} \chi_{\bar{V}}(g) \neq 0$, which is impossible since $\bar{V}$ is nontrivial and irreducible. Thus $\bar{V}$ is not self-dual as a $\bar{k} G$-module, and so $V$ is not self-dual as a $K G$-module.

Assume $\mathfrak{b}, \mathfrak{b}^{\prime}$ are both in $\operatorname{Sym}_{G}^{+}(V)$ or both in $\operatorname{Sym}_{G}^{-}(V)$. For each $\alpha \in K=$ $\operatorname{Aut}_{k G}(V)$, there is a unique $\bar{\alpha} \in K$ such that $\mathfrak{b}(\alpha(v), w)=\mathfrak{b}(v, \bar{\alpha}(w))$ for all $v, w \in$ $V$. By elementary considerations, $\alpha \mapsto \bar{\alpha}$ is a field automorphism $\psi \in \operatorname{Aut}(K)$, and $\psi^{2}=\operatorname{Id}_{K}$ since $\mathfrak{b}$ is symmetric or symplectic. If $\psi=\operatorname{Id}_{K}$, then $\mathfrak{b}$ factors through $V \otimes_{K} V$, so $\left(V \otimes_{K} V\right)^{G} \neq 0$, which is impossible since we just showed that $V \neq V^{*}$ as $K G$-modules. Thus $\psi$ is the automorphism of order two in $K$ (and $\left.\psi\right|_{k}=\operatorname{Id}_{k}$ ). Let $q$ be such that $\bar{\alpha}=\alpha^{q}$ for $\alpha \in K$; thus $|K|=q^{2}$.

Since $\mathfrak{b}$ and $\mathfrak{b}^{\prime}$ are nondegenerate, there is $\alpha \in \operatorname{Aut}_{k G}(V)=K^{\times}$such that $\mathfrak{b}^{\prime}(v, w)=\mathfrak{b}(\alpha(v), w)$ for each $v, w$. Also, $\bar{\alpha}=\alpha$, since $\mathfrak{b}^{\prime}$ and $\mathfrak{b}$ are both symmetric or both symplectic, so $\alpha^{q-1}=1$. Hence there is $\beta \in K$ such that $\alpha=\beta^{q+1}=\beta \bar{\beta}$, and so $\mathfrak{b}^{\prime}(v, w)=\mathfrak{b}(\beta(v), \beta(w))$ for each $v, w$. This proves (b) in this case.

If $\operatorname{char}(k) \neq 2$, then choose $\gamma \in K^{\times}$such that $\bar{\gamma} / \gamma=\gamma^{q-1}=-1$. Define $\mathfrak{b}^{\prime \prime}$ by setting $\mathfrak{b}^{\prime \prime}(v, w)=\mathfrak{b}(\gamma(v), w)$. Then $\mathfrak{b}^{\prime \prime} \in \operatorname{Sym}_{G}^{\mp}(V)$ if $\mathfrak{b} \in \operatorname{Sym}_{G}^{ \pm}(V)$, so both of these sets are nonempty. This finishes the proof of the lemma when $V$ is irreducible.

Now assume $V$ is a direct sum of nontrivial self-dual irreducible $k G$-modules. We claim that each form in $\operatorname{Sym}_{G}^{ \pm}(V)$ splits as an orthogonal direct sum of forms on simple summands of $V$. Assume otherwise, and let $(V, \mathfrak{b})$ be a minimal counterexample. Then $\left.\mathfrak{b}\right|_{W \times W}=0$ for each irreducible $W \subseteq V$. Fix irreducible submodules $W_{1}, W_{2} \subseteq V$ such that $W_{2} \oplus W_{1}^{\perp}=V$. Choose $\mathfrak{b}^{\prime} \in \operatorname{Sym}_{G}^{ \pm}\left(W_{2}\right)$ of the same type as $\mathfrak{b}$ (symmetric or antisymmetric), and define $\varphi: W_{1} \cong W_{2}^{*}$ and $\psi: W_{2} \cong W_{2}^{*}$ by setting $\varphi(v)(w)=\mathfrak{b}(v, w)$ and $\psi(v)(w)=\mathfrak{b}^{\prime}(v, w)$. Set $K=\operatorname{End}_{k G}\left(W_{2}\right)$, let $(\alpha \mapsto \bar{\alpha}) \in \operatorname{Aut}(K)$ be as above, and choose $\alpha \in K$ such that $\alpha+\bar{\alpha}=1$. Define $\rho: W_{2} \longrightarrow V$ by setting $\rho(v)=\alpha(v)+\varphi^{-1} \psi(v)$. Then for all $v, w \in W_{2}$,

$$
\begin{aligned}
\left(\left.\mathfrak{b}\right|_{W_{i} \times W_{i}}=0\right) \quad \mathfrak{b}(\rho(v), \rho(w)) & =\mathfrak{b}\left(\alpha(v), \varphi^{-1} \psi(w)\right)+\mathfrak{b}\left(\varphi^{-1} \psi(v), \alpha(w)\right) \\
& =\mathfrak{b}^{\prime}(\alpha(v), w)+\mathfrak{b}^{\prime}(v, \alpha(w)) \\
& =\mathfrak{b}^{\prime}(\alpha(v), w)+\mathfrak{b}^{\prime}(\bar{\alpha}(v), w)=\mathfrak{b}^{\prime}(v, w) .
\end{aligned}
$$


Thus $\mathfrak{b}$ does not vanish on $\rho\left(W_{2}\right)$, contradicting the original assumption. We conclude that $\mathfrak{b}$ splits as an orthogonal direct sum of forms on simple summands of $V$, and hence that the lemma holds for $V$ since it holds for its simple summands.

Step 2: Assume that none of the irreducible $k G$-summands of $V$ is self-dual (but $V$ is). Fix $\mathfrak{b} \in \operatorname{Sym}_{G}^{ \pm}(V)$. If $W_{1} \subseteq V$ is an irreducible summand, then $\left.\mathfrak{b}\right|_{W_{1} \times W_{1}}=0$ since $W_{1} \nsucceq W_{1}^{*}$. Choose a $k G$-summand $W_{2}$ complementary to $W_{1}^{\perp} \supseteq W_{1}$; thus $\mathfrak{b}$ is nondegenerate on $W_{1} \oplus W_{2}$. By continuing this procedure on $\left(W_{1} \oplus W_{2}\right)^{\perp}$, we get an orthogonal decomposition

$$
(V, \mathfrak{b})=\left(W_{1} \oplus W_{2}, \mathfrak{b}_{1}\right) \oplus \cdots \oplus\left(W_{2 r-1} \oplus W_{2 r}, \mathfrak{b}_{r}\right),
$$

where each $W_{i}$ is irreducible and each $\mathfrak{b}_{i}$ is a (symmetric or symplectic) hyperbolic form with respect to this decomposition. Since each such form is determined by isomorphisms $W_{2 i-1} \stackrel{\cong}{\longrightarrow} W_{2 i}^{*}$, any two such forms are isomorphic.

Since $V \cong V^{*}$, we can write $V=\bigoplus_{i=1}^{2 r} W_{i}$ where $W_{2 i-1} \cong W_{2 i}^{*}$, and hence $\operatorname{Sym}_{G}^{ \pm}(V)$ are both nonempty.

Lemma A.1 now allows us, for certain pairs of classical groups $H<G$, to show that $\mathcal{F}_{p}(H)$ is a full subcategory of $\mathcal{F}_{p}(G)$. In what follows, $G O_{n}^{ \pm}(q)$ denotes the full orthogonal group on $\mathbb{F}_{q}^{n}$.

Lemma A.2. Fix an odd prime $p$, and a prime power $q$ which is prime to $p$. Each of the following inclusions $H<G$ of finite groups induces an inclusion of $\mathcal{F}_{p}(H)$ as a full subcategory in $\mathcal{F}_{p}(G)$.

(a) $G L_{n}(q)<G L_{n+1}(q)$ for all $n \geq 2$.

(b) $G L_{n}(q)<G L_{n}\left(q^{k}\right)$ for all $n \geq 2$ and all $k>1$.

(c) $S p_{2 n}(q)<G L_{2 n}(q)$ for all $n$.

(d) $G O_{n}^{ \pm}(q)<G L_{n}(q)$ for all $n \geq 2$.

Proof. In each case $H<G$, we claim, for each $p$-group $P$, that $\operatorname{Rep}(P, H)$ injects into $\operatorname{Rep}(P, G)$. This follows from Lemma A.1 in cases (c) and (d) (applied to representations of $P$ ), and from semisimplicity and the unique decomposition of $\mathbb{F}_{q}[P]$-modules in cases (a) and (b). (Note that for any $\mathbb{F}_{q}[P]$-module $V, \mathbb{F}_{q^{k}} \otimes_{\mathbb{F}_{q}} V \cong$ $V^{k}$ as $\mathbb{F}_{q}[P]$-modules.) The lemma now follows from Proposition 1.6 .

Whenever $H_{1}, H_{2}<G$ are inclusions of finite groups which make $\mathcal{F}_{p}\left(H_{1}\right)$ and $\mathcal{F}_{p}\left(H_{2}\right)$ into full subcategories of $\mathcal{F}_{p}(G)$, and the Sylow $p$-subgroups of $H_{1}$ and $H_{2}$ are $G$-conjugate, then $\mathcal{F}_{p}\left(H_{1}\right) \simeq \mathcal{F}_{p}\left(H_{2}\right)$. Using this, Lemma A.2 leads to the following examples of isotypically equivalent fusion systems.

Proposition A.3. For any odd prime $p$, and any prime power $q$ prime to $p$, the following pairs of fusion systems are isotypically equivalent.

(a) $\mathcal{F}_{p}\left(S O_{2 n+1}(q)\right) \simeq \mathcal{F}_{p}\left(S p_{2 n}(q)\right)$ for all $n \geq 1$.

(b) $\mathcal{F}_{p}\left(G O_{2 n}^{ \pm}(q)\right) \simeq \mathcal{F}_{p}\left(S O_{2 n+1}(q)\right)$ if $q^{n} \not \equiv \mp 1(\bmod p)$.

(c) $\mathcal{F}_{p}\left(S O_{2 n-1}(q)\right) \simeq \mathcal{F}_{p}\left(S O_{2 n}^{ \pm}(q)\right)$ if $q^{n} \not \equiv \pm 1(\bmod p)$.

(d) $\mathcal{F}_{p}\left(S p_{2 n}(q)\right) \simeq \mathcal{F}_{p}\left(S L_{2 n}(q)\right)$ for each $n \geq 1$ if $\operatorname{ord}_{p}(q)$ is even.

(e) $\mathcal{F}_{p}\left(S O_{2 n+1}(q)\right) \simeq \mathcal{F}_{p}\left(S L_{2 n+1}(q)\right)$ for each $n \geq 1$ if $\operatorname{ord}_{p}(q)$ is even. 
(f) $\mathcal{F}_{p}\left(S p_{2 n}(q)\right) \simeq \mathcal{F}_{p}\left(S p_{2 n}\left(q^{2}\right)\right)$ for each $n \geq 1$ if $\operatorname{ord}_{p}(q) \equiv 2(\bmod 4)$.

(g) $\mathcal{F}_{p}\left(G L_{s m}(q)\right) \simeq \mathcal{F}_{p}\left(G L_{s m+i}(q)\right)$ if $s=\operatorname{ord}_{p}(q)>1, m \geq 1$, and $0<i<s$.

Proof. By Lemma A.1, each $p$-subgroup of $S p_{2 n}(q)$ is $G L_{2 n+1}(q)$-conjugate to a subgroup of $S O_{2 n+1}(q)$ and vice versa. Hence the Sylow $p$-subgroups of $S p_{2 n}(q)$ and $G L_{2 n+1}(q)$ are conjugate in $G L_{2 n+1}(q)$, which by LemmaA.2 implies that their $p$-fusion systems are isotypically equivalent. This proves (a).

The other cases follow by comparing the orders of the groups in question and applying Lemma A.2. For example, (b) and (c) follow from the standard order formulas

$$
\begin{aligned}
& {\left[S O_{2 n+1}(q): S O_{2 n}^{ \pm}(q)\right]=q^{n}\left(q^{n} \pm 1\right),} \\
& {\left[S O_{2 n}^{ \pm}(q): S O_{2 n-1}(q)\right]=q^{n-1}\left(q^{n} \mp 1\right) .}
\end{aligned}
$$

Note that if $H<K<G$ induces an isotypical equivalence $\mathcal{F}_{p}(H) \simeq \mathcal{F}_{p}(G)$, then $\mathcal{F}_{p}(H)$ is a full subcategory of $\mathcal{F}_{p}(K)$, and hence $\mathcal{F}_{p}(H) \simeq \mathcal{F}_{p}(K)$. Also, $S O_{2 n+1}(q)$ and $G O_{2 n+1}(q) \cong S O_{2 n+1}(q) \times\{ \pm \mathrm{Id}\}$ always have the same fusion system at odd primes.

\section{Appendix B. Homotopy fiXed points AND DOUble MAPPING TELESCOPES}

We give here short proofs of two elementary results needed in Section 2 .

Lemma B.1. Assume $X, Y$ are spaces, $\alpha: X \stackrel{\simeq}{\longrightarrow} X$ and $\beta: Y \stackrel{\simeq}{\longrightarrow} Y$ are homotopy equivalences, and $f: X \stackrel{\simeq}{\longrightarrow} Y$ is a homotopy equivalence such that $f \circ \alpha \simeq$ $\beta \circ f$. Then $X^{h \alpha} \simeq Y^{h \beta}$.

Proof. For any map $f: X \longrightarrow Y$ and any homotopy $F: X \times I \longrightarrow Y$ from $f \alpha$ to $\beta f$ (i.e., $F(-, 0)=f \alpha$ and $F(-, 1)=\beta f$ ), define

$$
\Phi_{f, F}: X^{h \alpha} \longrightarrow Y^{h \beta}
$$

by setting, for each $\phi \in X^{h \alpha}$,

$$
\Phi_{f, F}(\phi)(t)= \begin{cases}f(\phi(2 t)) & \text { if } t \leq \frac{1}{2}, \\ F(\phi(0), 2 t-1) & \text { if } t \geq \frac{1}{2} .\end{cases}
$$

These maps have the following properties:

(a) Assume $Z$ is a third space, with homotopy equivalence $\gamma: Z \stackrel{\simeq}{\longrightarrow} Z$. For any pair of maps $X \stackrel{f}{\longrightarrow} Y \stackrel{g}{\longrightarrow} Z$, and any pair of homotopies $F$ from $f \alpha$ to $\beta f$ and $G$ from $g \beta$ to $\gamma g$, there is a homotopy $H$ from $g f \alpha$ to $\gamma g f$ such that $\Phi_{g, G} \circ \Phi_{f, F} \simeq \Phi_{g f, H}$. For example, we can set

$$
H(x, t)= \begin{cases}g(F(x, 2 t)) & \text { if } t \leq \frac{1}{2}, \\ G(f(x), 2 t-1) & \text { if } t \geq \frac{1}{2} .\end{cases}
$$

(b) For any $f$ and $F$ as above, and any $f^{\prime} \simeq f$, there is a homotopy $F^{\prime}$ from $f \alpha$ to $\beta f$ such that $\Phi_{f^{\prime}, F^{\prime}} \simeq \Phi_{f, F}$. For example, if $R: X \times I \longrightarrow Y$ is the homotopy, where $R(-, 0)=f$ and $R(-, 1)=f^{\prime}$, we can define $F^{\prime}$ by setting

$$
F^{\prime}(x, t)= \begin{cases}R(\alpha(x), 1-3 t) & \text { if } t \leq \frac{1}{3} \\ F(x, 3 t-1) & \text { if } \frac{1}{3} \leq t \leq \frac{2}{3} \\ \beta(R(x, 3 t-2)) & \text { if } \frac{2}{3} \leq t\end{cases}
$$


We leave it to the reader to draw the picture and construct the homotopy from $\Phi_{f, F}$ to $\Phi_{f^{\prime}, F^{\prime}}$.

(c) Let $F$ be any homotopy from $\alpha$ to itself, and let $\bar{F}$ be the homotopy $\bar{F}(x, t)=$ $(x, 1-t)$. Then $\Phi_{\operatorname{Id}_{X}, F}$ is a homotopy equivalence with homotopy inverse $\Phi_{\operatorname{Id}_{X}, \bar{F}} \cdot$

Now assume $f: X \longrightarrow Y$ is a homotopy equivalence with homotopy inverse $g$. Then $g \beta \simeq \alpha g$. Choose homotopies $F$ from $f \alpha$ to $\beta f$ and $G$ from $g \beta$ to $\alpha g$. By (a) and (b), there are homotopies $H_{1}$ and $H_{2}$ such that $\Phi_{g, G} \circ \Phi_{f, F} \simeq \Phi_{\operatorname{Id}_{X}, H_{1}}$ and $\Phi_{f, F} \circ \Phi_{g, G} \simeq \Phi_{\operatorname{Id}_{Y}, H_{2}}$. These composites are homotopy equivalences by (c), and so $\Phi_{f, F}$ is a homotopy equivalence.

Recall that we define the mapping telescope of $\alpha: X \longrightarrow X$ to be the space

$$
\operatorname{Tel}(\alpha)=(X \times I \times \mathbb{Z}) / \sim, \quad \text { where } \quad(x, 0, n) \sim(\alpha(x), 1, n-1) \quad \forall x \in X, n \in \mathbb{Z} .
$$

Lemma B.2. For any space $X$ and any homotopy equivalence $\alpha: X \longrightarrow X$, the inclusion of $X$ in $\operatorname{Tel}(\alpha)$ which sends $x \in X$ to $[x, 0,0]$ is a homotopy equivalence.

Proof. For each $n \geq 0$, let $\operatorname{Tel}_{n}(\alpha) \subseteq \operatorname{Tel}(\alpha)$ be the image of $(X \times I \times\{-n, \ldots, n\})$ in $\operatorname{Tel}(\alpha)$. The inclusion of $\operatorname{Tel}_{n}(\alpha)$ into $\operatorname{Tel}_{n+1}(\alpha)$ is a homotopy equivalence since $\alpha$ is, and it has the homotopy extension property since it has a "mapping cylinder neighborhood" in the sense of [Ha, Example 0.15]. Hence $\operatorname{Tel}_{n}(\alpha)$ is a strong deformation retract of $\operatorname{Tel}_{n+1}(\alpha)$ by $\mathrm{Ha}$, Corollary 0.20]. One can now take the "composite" of these strong deformation retractions, to prove that $\operatorname{Tel}_{0}(\alpha)$ (and hence $X)$ is a strong deformation retract of $\operatorname{Tel}(\alpha)$.

\section{REFERENCES}

[AGMV] K. Andersen, J. Grodal, J. Møller, and A. Viruel, The classification of p-compact groups for $p$ odd, Annals of Math. (2) 167 (2008), 95-210. MR.2373153 (2009a:55012)

[A] M. Aschbacher, Finite Group Theory, Cambridge Univ. Press (1986). MR895134 (89b:20001)

[Bor] A. Borel, Sur la cohomologie des espaces fibrés principaux et des espaces homogènes de groupes de Lie compacts, Annals of Math. (2) 57 (1953), 115-207. MR0051508 $(14: 490 \mathrm{e})$

[BK] A. Bousfield and D. Kan, Homotopy limits, completions and localizations, 2nd ed., Lecture Notes in Mathematics, vol. 304 (1987). MR0365573 (51:1825)

[Br] G. Bredon, Equivariant cohomology theories, Lecture Notes in Mathematics 34, Springer-Verlag (1967). MR0214062 (35:4914)

[BtD] T. Bröcker and T. tom Dieck, Representations of compact Lie groups, Springer-Verlag (1985). MR781344 (86i:22023)

[BCGLO2] C. Broto, N. Castellana, J. Grodal, R. Levi, and B. Oliver, Extensions of p-local finite groups. Trans. Amer. Math. Soc. 359 (2007), 3791-3858. MR.2302515 (2008i:55013)

[BM] C. Broto and J. Møller, Chevalley p-local finite groups, Algebr. Geom. Topology 7 (2007), 1809-1919. MR2366180 (2009g:55017)

[DW] W. Dwyer and C. Wilkerson, Homotopy fixed-point methods for Lie groups and finite loop spaces, Ann. of Math. (2) 139 (1994), 395-442. MR1274096 (95e:55019)

[FM] P. Fong and J. Milgram, On the geometry and cohomology of the simple groups $G_{2}(q)$ and ${ }^{3} D_{4}(q)$, Group representations: cohomology, group actions and topology, Proc. Symp. Pure Maths. 63 (1998), 221-244. MR1603163 (99f:20088)

[Fr] E. Friedlander, Étale homotopy of simplicial schemes, Annals of Mathematics Studies, vol. 104, Princeton University Press (1982). MR676809 (84h:55012)

[Go] D. Gorenstein, Finite groups, Harper \& Row (1968) MR0231903 (38:229) 
[GLS3] D. Gorenstein, R. Lyons, and R. Solomon, The classification of the finite simple groups, nr. 3, Amer. Math. Soc. Surveys and Monogr. 40 \#3 (1997). MR.1490581 (98j:20011)

[GR] R. Griess and A. Ryba, Embeddings of $P G L_{2}(31)$ and $S L_{2}(32)$ in $E_{8}(\mathbb{C})$, Duke Math. J. 94 (1998), 181-211. MR1635916 (2000a:20106)

[Ha] A. Hatcher, Algebraic Topology, Cambridge University Press (2002). MR.1867354 (2002k:55001)

[JMO] S. Jackowski, J. McClure, and B. Oliver, Self-homotopy equivalences of classifying spaces of compact connected Lie groups, Fund. Math. 147 (1995), 99-126. MR.1341725 (96f:55009)

[Kl1] P. Kleidman, The maximal subgroups of the Steinberg triality groups ${ }^{3} D_{4}(q)$ and of their automorphism groups, J. Algebra 115 (1988), 182-199. MR937609 (89f:20024)

[K12] P. Kleidman, The maximal subgroups of the Chevalley groups $G_{2}(q)$ with $q$ odd, the Ree groups ${ }^{2} G_{2}(q)$, and their automorphism groups, J. Algebra 117 (1988), 30-71. MR 955589 (89j:20055)

[MP] J. Martino and S. Priddy, Unstable homotopy classification of $B G_{p}^{\wedge}$, Math. Proc. Cambridge Philos. Soc. 119 (1996), 119-137. MR.1356164 (96i:55025)

[Mi] J. Milgram, On the geometry and cohomology of the simple groups $G_{2}(q)$ and ${ }^{3} D_{4}(q)$ : II, Group representations: cohomology, group actions and topology, Proc. Symp. Pure Math. 63 (1998), 397-418. MR 1603199 (99f:20089)

[Ms] G. Mislin, On group homomorphisms inducing mod-p cohomology isomorphisms, Comment. Math. Helv. 65 (1990), 454-461. MR1069820 (92a:20059)

[Ml] J. Møller, N-determined 2-compact groups. I, Fundamenta Math. 195 (2007), 11-84. MR 2314074 (2008m:55013)

[MN] J. Møller and D. Notbohm, Centers and finite coverings of finite loop spaces, J. Reine Angew. Math. 456 (1994), 99-133. MR1301453 (95j:55029)

[O1] B. Oliver, Equivalences of classifying spaces completed at odd primes, Math. Proc. Camb. Phil. Soc. 137 (2004), 321-347. MR2092063 (2006g:55010)

[O2] B. Oliver, Equivalences of classifying spaces completed at the prime two, Amer. Math. Soc. Memoirs 848 (2006). MR2203209 (2007c:55014)

[Se] J.-P. Serre, Linear representations of finite groups, Springer-Verlag (1977). MR $0450380(56: 8675)$

[ST] G. Shephard and J. Todd, Finite unitary reflection groups, Canadian J. Math. 6 (1954), 274-304. MR0059914(15:600b)

Departament de Matemàtiques, Universitat Autònoma de Barcelona, E-08193 BelLATERRA, SPAIN

E-mail address: broto@mat.uab.es

Matematisk Institut, Universitetsparken 5, DK-2100 København, Denmark

E-mail address: moller@math.ku.dk

LAGa, Institut Galilée, Av. J-B Clément, F-93430 Villetaneuse, France

E-mail address: bobol@math.univ-paris13.fr 